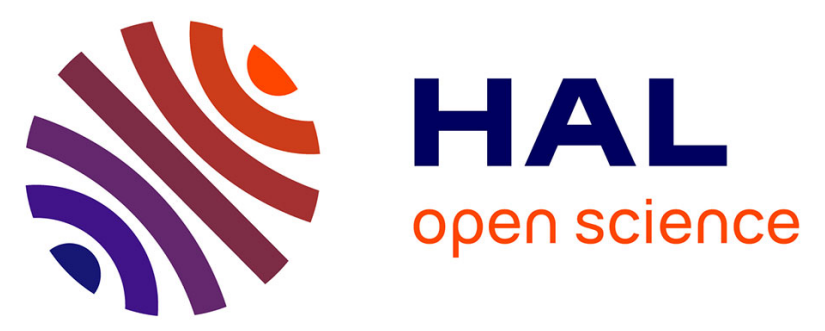

\title{
Construction of 3D MR image-based computer models of pathologic hearts, augmented with histology and optical fluorescence imaging to characterize action potential propagation
}

Mihaela Pop, Maxime Sermesant, Garry Liu, Jatin Relan, Tommaso Mansi, Alan Soong, Jean-Marc Peyrat, Michael V. Truong, P. Fefer, Elliot R. Mcveigh, et al.

\section{- To cite this version:}

Mihaela Pop, Maxime Sermesant, Garry Liu, Jatin Relan, Tommaso Mansi, et al.. Construction of 3D MR image-based computer models of pathologic hearts, augmented with histology and optical fluorescence imaging to characterize action potential propagation. Medical Image Analysis, 2012, 16 (2), pp.505-523. hal-00813859

\section{HAL Id: hal-00813859 \\ https://hal.inria.fr/hal-00813859}

Submitted on 3 May 2013

HAL is a multi-disciplinary open access archive for the deposit and dissemination of scientific research documents, whether they are published or not. The documents may come from teaching and research institutions in France or abroad, or from public or private research centers.
L'archive ouverte pluridisciplinaire HAL, est destinée au dépôt et à la diffusion de documents scientifiques de niveau recherche, publiés ou non, émanant des établissements d'enseignement et de recherche français ou étrangers, des laboratoires publics ou privés. 


\title{
Construction of 3D MR image-based computer models of pathologic hearts, augmented with histology and optical fluorescence imaging to characterize action potential propagation
}

\author{
Mihaela Pop ${ }^{1}$, Maxime Sermesant ${ }^{2}$, Garry Liu ${ }^{1}$, Jatin Relan ${ }^{2}$, Tommaso Mansi ${ }^{3}$, Alan Soong ${ }^{1}$, Jean-Marc Peyrat ${ }^{4}$, Michael V Truong ${ }^{3}$, \\ Paul Fefer ${ }^{5}$, Elliot R McVeigh ${ }^{6}$, Herve Delingette ${ }^{2}$, Alexander J Dick ${ }^{5}$, Nicholas Ayache ${ }^{2}$ and Graham A Wright ${ }^{1}$ \\ ${ }^{1}$ Dept. of Medical Biophysics and ${ }^{5}$ Cardiology, University of Toronto, Sunnybrook Research Institute, Canada \\ ${ }^{2}$ INRIA - Asclepios project, Sophia Antipolis, France; ${ }^{3}$ Siemens Corporate Research, Princeton, USA \\ ${ }^{4}$ Siemens Molecular Imaging, Oxford, UK \\ ${ }^{6}$ Department of Biomedical Engineering, Johns Hopkins Medical School, Baltimore, Maryland, USA
}

Corresponding author:

Mihaela Pop

mihaela.pop@utoronto.ca

Address:

2075 Bayview Avenue, room SG-18

Sunnybrook Research Institute

Department of Medical Biophysics

University of Toronto

M4N 3M5

Tel: $1-416-4806100$ ext 7129 


\section{Abstract:}

Cardiac computer models can help us understand and predict the propagation of excitation waves (i.e., action potential, AP) in healthy and pathologic hearts. Our broad aim is to develop accurate 3D MR image-based computer models of electrophysiology in large hearts (translatable to clinical applications) and to validate them experimentally. The specific goals of this paper were to match models with maps of the propagation of optical AP on the epicardial surface using large porcine hearts with scars, estimating several parameters relevant to macroscopic reaction-diffusion electrophysiological models. We used voltage-sensitive dyes to image AP in large porcine hearts with scars ( 3 specimens had chronic myocardial infarct, and 3 had radiofrequency RF acute scars). We first analyzed the main AP waves' characteristics: duration (APD) and propagation under controlled pacing locations and frequencies as recorded from 2D optical images. We further built 3D MR image-based computer models that have information derived from the optical measures, as well as morphologic MRI data (i.e., myocardial anatomy, fiber directions and scar definition). The scar morphology from MR images was validated against corresponding whole-mount histology. We also compared the measured 3D isochronal maps of depolarization to simulated isochrones (the latter replicating precisely the experimental conditions), performing model customization and 3D volumetric adjustments of the local conductivity. Our results demonstrated that mean APD in the border zone (BZ) of the infarct scars was reduced by 13\% (compared to 318 ms measured in normal zone, NZ), but APD did not change significantly in the thin BZ of the ablation scars. A generic value for velocity ratio (1:2.7) in healthy myocardial tissue was derived from measured values of transverse and longitudinal conduction velocities relative to fibers direction $(22 \mathrm{~cm} / \mathrm{s}$ and $60 \mathrm{~cm} / \mathrm{s}$, respectively). The model customization and 3D volumetric adjustment reduced the differences between measurements and simulations; for example, from one pacing location, the adjustment reduced the absolute error in local depolarization times by a factor of 5 (i.e., from 58 $\mathrm{ms}$ to $11 \mathrm{~ms}$ ) in the infarcted heart, and by a factor of 6 (i.e., from $60 \mathrm{~ms}$ to $9 \mathrm{~ms}$ ) in the heart with the RF scar. Moreover, the sensitivity of adjusted conductivity maps to different pacing locations was tested, and the errors in activation times were found to be of approximately 10-12 ms independent of pacing location used to adjust model parameters, suggesting that any location can be used for model predictions.

Keywords: cardiac computer models; MRI; optical imaging; electrophysiology 


\section{Introduction}

Cardiac computer models can be utilized to study normal propagation of action potential (AP) and potential inducibility of arrhythmic events, with the most dangerous manifestation of arrhythmias being ventricular tachycardia (VT) and ventricular fibrillation (VF) (Janse and Wit 1989, Kleber and Rudy 2004). Theoretical predictions of AP propagation play a major role in electrophysiology since they can complement clinical observations (limited to surfacic measures) helping one understand inducibility of reentrant VT/VF, and they can give mechanistic insights into the generation of VF and defibrillation mechanisms. In particular, attention has been recently given to 3D MR image-based models built from high resolution scans of hearts with myocardial infarction (Vadakkumpadan et al 2009, Vigmond et al 2009), a major cause of sudden death in the industrialized world (Martinez-Rubio et al 1999). These sophisticated in silico computer models of the heart could not only advance the basic cardiac research field, but also help clinicians tailor radiofrequency ablation (RFA) therapy, assist in the implantation of defibrillator devices and optimize cardiac resynchronization therapies (Trayanova 2006, 2009). However, before integrating these mathematical models into realistic clinical platforms, they have to be validated experimentally with a sufficient level of detail and accuracy (Clayton 2001, Hunter et al 2008). In addition, regardless of the species modeled, such validations are often hampered by the degree of complexity specific to multi-scale models as well as the large number of variables involved in the mathematical equations.

Research is needed to enrich the current cardiac experimental databases and to perform model customization from electrophysiological data/measures, particularly for large animal hearts that are the most relevant to human clinical applications. Important tasks include: 1) the choice of appropriate imaging modality that provides sufficient detail of anatomy, fiber direction and morphology (scar) to construct the 3D heart model, including histological validation of scar heterogeneities; 2) the choice of appropriate mathematical model whose output parameters can be compared at a spatiotemporal scale replicating experiments performed under precisely controlled conditions; 3) the selection of a suitable experimental technique for electrophysiological measures; and 4) model customization and adjustment of the input parameters.

One imaging modality of choice is MRI, currently used to evaluate cardiac disease in preclinical studies and routine clinical examinations. For instance, late-enhancement MRI (using contrast agents) is known to be a powerful tool used to characterize infarct heterogeneities associated with reentrant VT in patients with myocardial infarction (Yan et al 2006, Schmidt et al 
2007), but the current 5-8mm slice thickness used can introduce partial volume effects. In addition, the right ventricle wall cannot be well visualized and segmented; therefore, the accuracy in reconstructing a 3D heart in-vivo model could be severely affected. In an animal model of macro-reentrant VT, the electrophysiological properties of tissue were correlated with the contrast-enhanced MR signal (i.e., anatomic identification of dense scar and the border zone) obtained ex vivo, with sub-mm resolution (Ashikaga et al 2007). Unfortunately, these studies lack the evaluation of fiber direction and the local quantification of anisotropy within the infarction area, which is crucial to the modelling of wave propagation in myocardial tissue. Alternatively, other ex-vivo MR studies have explored non-contrast techniques like diffusion-tensor (DT) MRI, which are particularly useful in scar identification and reconstruction of fiber orientation (Wu et al 2007). In order to accurately determine the extent of dense, collagenous scar in chronic infarcts, one can compute maps of fractional anisotropy (FA) and/or apparent diffusion coefficient (ADC), and, based on the MR signal heterogeneity, these maps can be further segmented into healthy tissue, border zone and core scar zone. Using such high-resolution maps, 3D cardiac computer models have been constructed so far for rabbit, dog, and porcine hearts (Bishop et al 2010, Vadakkumpadan et al 2010, Pop et al 2009b) and used for different simulation purposes.

Cardiac multi-scale and multi-dimensional mathematical models give unprecedented detail as they integrate structural and functional information from sub-cellular levels, to slabs of myocardial tissue and whole organ (Gavaghan et al 2006, Austin et al 2006, Hunter and Nielsen 2005, Hunter et al 2008, Prassl et al 2009, Niederer 2011); however, one has to consider the level of detail of the model when considering implementation in a clinical setting where computational time is an issue. Moreover, certain applications might not justify the use of sophisticated equations and super-computers. The fastest and simplest numerical model is based on the eikonal equations (Keener and Sneyd 1998) which compute wavefront propagation; the result can be compared with clinically observed surfacic measures of depolarization isochrones; however, this model lacks refractory properties of the myocardium. At an intermediate level of complexity are the monodomain equations based on reaction-diffusion phenomena (Aliev and Panfilov 1996b). In these models, the heart is modeled as a continuous medium (syncytium) and the solution for AP captures the main characteristics of the AP wave: duration, shape and upstroke. For simple applications, the monodomain model can be used to simulate normal and reentrant wave propagation in large hearts (Nash and Panfilov 2004, Aliev and Panfilov 1996a), requiring less than $1 \mathrm{~h}$ computational time (on an ordinary PC) to simulate $1 \mathrm{~s}$ of cardiac cycle (Sermesant et al 2006). This choice is also attractive for some clinical (Sermesant et al 2005), given that little 
difference $(2 \%)$ in the activation times was found compared to the bidomain model (Potse et al 2007), which requires 130 CPUs to simulate 1s of heart cycle.

Finally, the experimental technique should be appropriately selected to enable comparison between measurements and the outputs of the theoretical model, at the same spatio-temporal scale. The comparisons can be used to customize the model; that is, several parameters of interest can be estimated from measurements and used to adjust the model variables. At the organ level, except for monophasic action potential catheter measurements (Kim et al 2002, Hao et al 2004), the clinical electrophysiological techniques are limited to measurements of depolarization times (Schmitt et al 1999, Dukkipati et al 2008); moreover, all provide surfacic measurements only, primarily from the endocardium. An alternative is to study electrophysiology at the tissue level; optical fluorescence imaging (which uses fast voltage-sensitive dyes) provides accurate measurements of AP waves in explanted perfused hearts prepared under physiological conditions approximating those seen in vivo (Efimov et al 2004; Kay et al 2004, Banville et al 2004, Yang 2007, Nanthakumar et al 2007). Several optical studies demonstrated that the optical technique is also feasible for mapping action potential propagation in the presence of structural obstacles, which perturb normal propagation. For instance, epicardial propagation of AP (during pacing conditions or VT/VF) was mapped in rabbit and rat hearts with chronic infarcts (Li et al 2004, Mills et al 2005) and revealed changes in conduction velocity at the border zone of the infarct. Other studies mapped the AP propagation in the presence of macroscopic obstacles created by thermally damaging the tissue using cryoablation, high intensity focused ultrasound or laser energy (Juong et al 2006, Qu et al 2004, Pastore and Rosenbaum 2000, Girouard and Rosenbaum 2001). Using our experimental set-up (Pop et al 2007) we recently reported results obtained by mapping optical AP waves in large, healthy porcine hearts. We also performed 3D model construction from MRI scans of the same hearts and adjusted the associated model based on optical measurements (Pop et al 2009a).

In this paper, we present novel results obtained in experimental and theoretical studies for explanted porcine hearts with scars. In accordance with the diagram below (Figure 1), we map the optical AP propagation in pathologic hearts and build 3D cardiac computer models allowing monodomain formalism, specifically, reaction-diffusion equations proposed by Aliev and Panfilov (1996b). We further estimate model parameters from the comparison between the measured and computed activation maps, and observe the model's behaviour in response to stimulation at different locations and with various pacing frequencies. Specifically, we built 3D MR-imagebased models obtained from large porcine hearts with two different types of large scars: 1) large 
lesions generated by RF ablation; and 2) chronic infarct scars. We hypothesized that the first type is an unexcitable scar that will not propagate action potential due to myocytes' necrosis within the thermally coagulated scar; this corresponds to a simplified 3D heart model comprised of two zones: healthy (with normal conductivity) and scar (with zero conductivity). The second type of pathology (i.e., chronic infarct) was selected due to heterogeneities specific to the healing process, where dense collagenous scar and border zone (i.e., a mixture of necrotic and surviving myocytes with altered electrophysiological properties) give rise to a more complex cardiac electrical propagation.

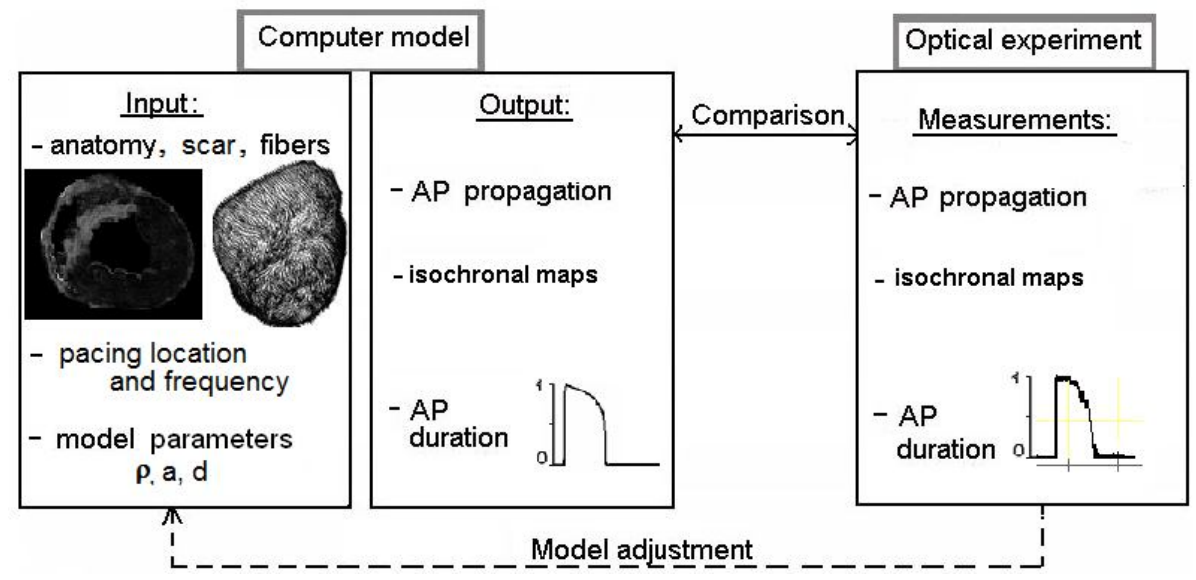

Fig. 1. Study overview - 3D MRI-based computer models of pathologic scars, together with comparison to and model customization from optical fluorescence measurements of action potential, AP.

The last goal was to demonstrate the potential for parameter estimation and model adjustment from experimental measures for a given stimulus pattern and subsequent prediction of response to different stimuli. To achieve the customization, we estimated several parameters (e.g., anisotropy ratio, AP duration and up-stroke) directly from 2D optical recordings; and also adjusted the 3D conductivity map (tuning the conduction velocity) by minimizing iteratively the error between simulated and measured depolarization times. Finally, to observe the influence of perturbation in fiber orientation, we generated synthetic fibers and studied their effect on the activation times compared with those obtained with the realistic directions calculated from DTI. 


\section{Materials and Methods}

We first performed the optical experiments of AP propagation on explanted hearts, and then used 3D MR scans to construct the heart model (i.e., the anatomy, fiber directions and scar). The computational mesh was generated from the anatomy scans and the simulations were performed with model parameters selected to reproduce exactly the experimental conditions, for instance stimulation at a given pacing frequency with a stimulus of certain duration and location. After estimating the main parameters (i.e., anisotropy ratio and AP duration) directly from 2D optical images, we performed further model customization and adjusted the local conductivity from maps of isochrones depolarization. We present below in detail the steps performed to build both the experimental and theoretical models, and associated analysis.

\subsection{Optical experiment}

Optical imaging was performed in 6 hearts explanted from swine weighing 37-42kg. Each animal was anesthetized, the chest opened, and the heart explanted in accordance with the animal research protocol guidelines approved at Sunnybrook Health Sciences Centre, Toronto, Canada. Two types of scars were created in various hearts as follows:

- In three hearts, large, acute RF ablation lesions were generated using an RF electrode operating at $460 \mathrm{kHz}$; these lesions were created in the left ventricle (LV) of the heart ex vivo;

- In three hearts, chronic myocardial infarcts (MIs), 4-weeks old at the excision time were studied; each infarction was generated in an anesthetized animal by a 90 -min coronary artery occlusion via a balloon catheter inserted either in the left anterior descending (LAD) or left circumflex (LCx), followed by reperfusion and recovery.

After excision, the aorta of each heart was rapidly cannulated and attached to a Langendorff ex vivo perfusion system. Throughout the experiments, the hearts were constantly perfused with an oxygenated mixture of blood and Tyrodes' solution $(95 \% \mathrm{O} 2$ and $5 \% \mathrm{CO} 2$; the ratio blood: solution was $1: 4)$ maintained at a temperature of $37.0 \pm 0.5^{\circ} \mathrm{C}$ and at a $\mathrm{pH}$ of $7.3 \pm 0.3$. The hearts were paced via a bipolar stimulating $\mathrm{Ag} / \mathrm{AglCl} 2$ electrode (E) coated with gold at the tip to avoid polarization effects. A square-wave stimulus of $5 \mathrm{~ms}$ duration was applied at different locations, and with 4 to 5 different frequencies, via a SD9 stimulator (GrassTelefactor, USA) operating in constant-voltage mode (the applied voltage during stimulation was about 3-4V). 
As in our previous optical study performed in healthy porcine hearts (Pop et al 2009a), a $20-\mathrm{ml}$ bolus of fluorescence dye solution ( $0.2 \mathrm{ml}$ di4-ANEPPS, Biotium Inc) was injected into the heart via the perfusion line. To suppress the motion artifacts associated with myocardial contraction, an electro-mechanical uncoupler (2,3-butanediome monoxime, Sigma Aldricht) was also injected in the perfusion line right after the dye. The fluorescent dye was excited with green light (530 $\pm 20 \mathrm{~nm}$ ) via 150-W halogen lamps (MHF G150LR, Moritek Corp, Japan), denoted "S" in Figure 2a. To avoid photo-bleaching, the lamps were controlled by shutters. The emitted signals from the fluorescing hearts were filtered through a high-pass filter (> $610 \mathrm{~nm})$ and captured by a high-speed dual-CCD system (MICAM02, BrainVision Inc. Japan) with 3.7-ms temporal resolution. The field of view of $184 \times 124$ pixels $(12 \times 8 \mathrm{~cm})$ yielded approximately $0.7 \mathrm{~mm}$ spatial resolution.

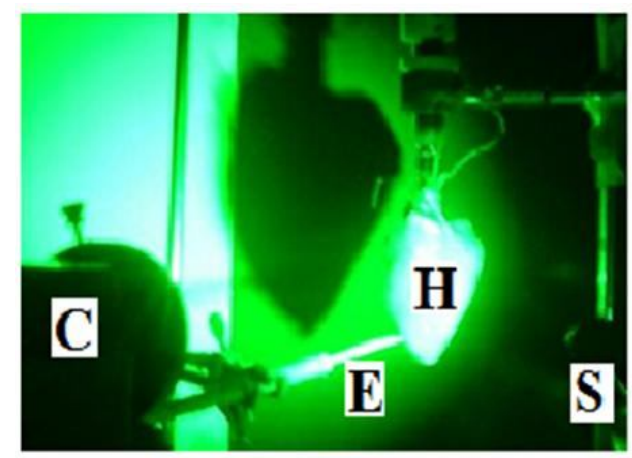

(a)
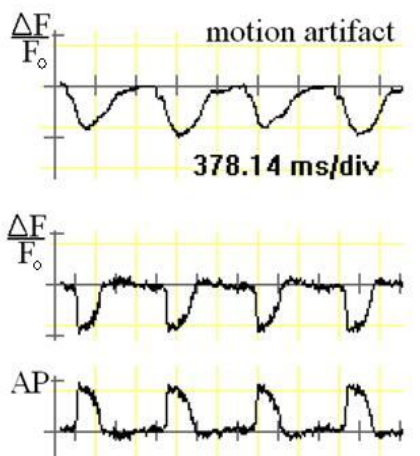

(b)

Fig. 2. (a) Snapshot of the optical experiment using MICAM02 cameras (C) to record epicardial action potential (AP) wave propagation from ex-vivo hearts attached to a Langendorff perfusion system. (b) Examples of waves recorded in the heart without the uncoupler (top) and after the uncoupler (bottom) has been distributed throughout the tissue; the relative loss of fluorescence signal $\Delta \mathrm{F} / \mathrm{F}=\left(\mathrm{F}-\mathrm{F}_{0}\right) / \mathrm{F}_{0}$ gives the change in transmembrane potential (i.e., AP). Note that $\Delta \mathrm{F} / \mathrm{F}$ and $\mathrm{AP}$ are in arbitrary units (a.u.) using $\mathrm{BV}$-Ana software.

The relative changes in the intensity of the fluorescence follow the changes in transmembrane potential. Figure $2 \mathrm{~b}$ shows examples of recorded optical fluorescence signal prior to injection of the uncoupler (top) during pacing at $1.1 \mathrm{~Hz}$; the signal was denoised using a spatialcubic filter and displayed with BV-Ana software (BrainVision, Japan). Further analysis of the AP waves was performed using custom built Matlab code that enabled normalization of the AP wave at each pixel, calculation of APD90 (APD at 90\% recovery), and averaging of results over the three hearts for each type of lesion. At each pixel, the optical traces as well as associated activation (depolarization time) and recovery (repolarization time) were detected using the first $(\mathrm{dF} / \mathrm{dt})$ and second $\left(\mathrm{d}^{2} \mathrm{~F} / \mathrm{dt}^{2}\right)$ derivatives of the fluorescence signal intensity versus time as per the 
method established by Efimov et al. (Efimov, 1994). This allowed us to verify (over selected regions of interest, ROI), the behaviour of rate-dependent properties of APD during pacing at various frequencies. Specifically, we analyzed the APD restitution curve during dynamic pacing (i.e., constant cycle length) performed in two hearts. These two hearts (one with an RF lesion and one with an infarct scar) were further used to build the 3D image-based models described in detail below. The optical images recorded by the CCD cameras from these two hearts were calibrated, rectified, and used to reconstruct the $3 \mathrm{D}$ surface of each heart according to the stereoscopic method described elsewhere, using custom built Matlab code developed by Chung et al (2006). The 3D optical stereo-surface of epicardium was further registered with the 3D surface of the MR image-based model as in (Pop et al 2007), via opaque markers visible in MR images, and using a rigid registration method implemented in Matlab (Horn, 1987). This step allowed us to project the measured isochronal maps onto each 3D heart mesh, similarly as presented in (Pop et al 2007 and 2009a).

\subsection{Magnetic Resonance Imaging, Heart Segmentation and Histology}

The pig ventricles were imaged using a 1.5-Tesla Signa GE MR scanner; the gross anatomy, fiber directions, fiducial markers, infarct, and infarct heterogeneity were all visualized in the MR images. As previously described (Pop et al 2007, 2009a), for determining the marker locations on the hearts, we used a 3D fast spin echo (FSE) sequence with the following MR parameters: TE = $60 \mathrm{~ms} ; \mathrm{TR}=700 \mathrm{~ms}$; slice thickness $=2 \mathrm{~mm}$; a $10 \mathrm{~cm} \mathrm{FOV}$; and 256x256 matrix acquisition yielding an in-plane resolution of $0.4 \times 0.4 \mathrm{~mm}$. The diffusion-weighted imaging sequence (Helm et al., 2005) was developed at the Laboratory of Cardiac Energetics (NIH/NHLBI, USA) and implemented on our research scanner, for which we used the following MR parameters: $\mathrm{TE}=30 \mathrm{~ms}$; TR=700ms; NEX=1; maximum $b$-value of approximately 600; and 7 directions for diffusion gradients, with the same FOV/matrix as was used in the FSE sequence.

For both hearts, the anatomy was extracted from the un-weighted images (i.e., $b=0$ ) and used to generate the volumetric mesh for the 3D computer model. DT images were used to reconstruct the fiber directions in a manner similar to our previous in healthy hearts.

Next, 3D volumetric ADC maps were computed from diffusion-weighted MR images using MedINRIA (ww-sop.inria.fr/asclepios/software/MedINRIA/); these 3D ADC maps were further used to segment the hearts. Specifically, the heart with the RF lesion was segmented into two zones: normal/healthy tissue and scar. The infarcted heart had three zones: healthy; dense 
infarct scar; and border zone. The latter corresponds to myocytes with viable channels but abnormal electrical activity. For partitioning the ventricular myocardium into the two or three morphologic zones, we employed an image segmentation tool developed in Matlab using the expectation-maximization (EM) algorithm (Van Leemput et al 2001), which does an expectation step where the likelihood of initial parameters given the existing data is calculated, then uses these statistics via a maximization step to search for new parameters values that would maximize this likelihood. In our case, the healthy myocardium tissue and scar are modeled by two Gaussian distributions based on histogram analysis. For the infarcted heart, for instance, voxels in the BZ of the scar, which do not extend farther than $8 \mathrm{~mm}$ away from the scar, are seen as outliers of the models. The algorithm is initialized using a two-class k-means method. During the EM loop, the means and variances of the two Gaussian distributions are estimated from the voxels classified as healthy and scar classes at the previous EM iteration (EM maximization step). Once the parameters are estimated, a belief value is computed. The belief quantifies how probable a voxel belongs to a given class, i.e., if it is below than a given threshold $(\lambda=0.5)$ for both tissue classes, the voxel is rejected, and it is considered as an outlier of the model (here, BZ). Prior knowledge about the BZ is implemented in the belief value. At a given stage of the EM algorithm, a distance map to the current estimation of the scar area is computed. Voxels farther than $5 \mathrm{~mm}$ from the scar are never rejected; they should belong to either scar or healthy tissue. A voxel close to the scar is rejected if its belief value is lower than $\lambda$ and half of its direct neighbors (6-connectivity) are also rejected. This simplified Markov spatial regularization aims to reduce the effects of image noise on the segmentation. Finally, the image is reclassified into the two classes plus the outliers (EM expectation step), resulting in three zones (NZ, BZ and scar). The algorithm loops until convergence. Similarly, for the heart with scar a simplified algorithm was implemented, since our assumption was that this heart has only two classes/zones (and no outliers for BZ). Finally, the segmented scar areas delineated by the EM algorithm were qualitatively compared to the areas of necrosis identified in histological slices.

For histological analysis, these two hearts were preserved in $10 \%$ neutral-buffered formalin for approximately 1 week to avoid shrinkage; then, right after the MR scan, histological processing was performed on selected slices ( $4 \mathrm{~mm}$ in thickness) cut to match the MRI short-axis views. Tissue deformation and rupture during dissection and slicing was minimized using a special whole-mount microtome cutting system (Clarke et al 2007). During the histological processing, the heart samples were dehydrated, embedded in paraffin, cut with a 5- $\mu \mathrm{m}$ slice thickness, fixed on glasses, and finally stained with Masson's Trichrome dye (in which healthy 
myocytes stain red, collagen fibers stain green, and nuclei stain black). The whole-mount slides were digitally scanned at 5-10 $\mu \mathrm{m}$ resolution and further analyzed using the Aperio-Image Scope software.

\subsection{Mathematical model and its implementation on the 3D mesh}

We used the two-variable reaction-diffusion mathematical model proposed by Aliev-Panfilov (1996b) of the heart to perform our simulations, extending the implementation previously detailed in (Sermesant et al 2006), to include restitution properties as presented in (Nash and Panfilov 2004). In this macroscopic model, described by the system of equations (1-2), we solved for $V$, the action potential, and $r$, the recovery variable contribution:

$$
\begin{aligned}
& \frac{\partial V}{\partial t}=\nabla \cdot(\tilde{D} \nabla V)-k V(V-a)(V-1)-r V+I_{\text {stim }} \\
& \frac{\partial r}{\partial t}=-\left(\varepsilon+\frac{\mu_{1} r}{\mu_{2}+V}\right)(k V(V-a-1)+r)
\end{aligned}
$$

where, except for $a$ and $k$, most of the reaction and recovery parameters were set as in Nash and Panafilov (2004), that is $\varepsilon=0.01, \mu_{1}=0.01$ and $\mu_{2}=0.3$. Generally, the term $I_{\text {stim }}$ represents the stimulating current necessary to produce a local depolarization that spreads from cell to cell through electrotonic diffusion; however, in our simulations, this is achieved by applying a square pulse of short duration (5ms), i.e., a constant voltage $V$ of maximum amplitude on a small area on the mesh. The fast-variable of the equation, $V$ is normalized (reaching a value of maximum amplitude "1" during excitation, followed by a slow recovery to the resting value, "0") and its waveform reproduced the shape, duration, and restitution of action potentials obtained from experiments in canine myocytes (Elharrar and Surawicz 1983).

The diffusion tensor $\tilde{D}=d\left(\begin{array}{lll}1 & 0 & 0 \\ 0 & \rho & 0 \\ 0 & 0 & \rho\end{array}\right)$ accounts for tissue diffusivity and anisotropy, and was written in the local orthonormal basis $(i, j, k)$ of the fiber direction, where $i$ is the direction parallel to the fiber. One parameter of interest is the normalized scalar diffusivity, $d$, which is often referred to as "bulk" or "pseudo-conductivity" (Ten Tusscher and Panfilov 2007, Moreau et al 2006, Clayton 2009); and is set to 1 for normal/healthy myocardial tissue. The conduction velocity is proportional to the square root of bulk conductance (Walton and Fozzard 1983, Kleber and Rudy 2004); thus, this coefficient $d$ determines the conduction velocity. Moreover, the other important parameter in the tensor $\tilde{D}$, is $\rho$, the anisotropy ratio, which is computed as the squared 
value of the ratio between the conduction velocity measured in the transverse and in the longitudinal direction (relative to the fibers). For instance, the velocity ratio is $1: 1$ for an isotropic medium and 1:3 for an electrical wave propagating twice as fast along versus across the fiber (thus, the latter gives a value $\rho=0.11$ ). Changes in this value result in variations in the propagation velocity of the depolarization front.

We solved the above reaction-diffusion equations for the transmembrane potential $(V)$ using the Finite Element Method, with an explicit Euler time integration scheme. The code was written in $\mathrm{C}++$ and used OpenGL libraries to display the results. The equations were solved over a cardiac surface mesh which was created from the anatomical images of the heart using classical segmentation algorithms (thresholding, mathematical morphology, marching cubes) followed by a tetrahedrization of the 3D volume with the TetMesh-GHS3D package (INRIA, France). For each vertex, the assigned fiber direction is the principal eigenvector, as derived from the DTI. For the simulations presented in this study, we used a computational time step of $5 \times 10^{-5} \mathrm{~s}$. The simulation time for $0.8 \mathrm{~s}$ of the cardiac cycle on a mesh of approximately 180,000 elements, was about 25 min on an Intel ${ }^{\circledR}$ Core (TM) 2 Duo CPU $1.83 \mathrm{GHz}$, with 4GB of RAM.

In simulations, the excitation starts from a few selected vertices corresponding to the precise location of the electrode tip in the optical experiment (determined after projection of optical image onto the 3D mesh). For the boundary conditions, as this is an isolated heart model, we assumed that no currents flow out of the myocardium; thus, a Neumann boundary condition was imposed to represent this. As a result, the wave travels parallel to the surface. Thus, the 2D and 3D speed vectors coincide on the surface. Furthermore, depolarization velocity vectors were computed per triangle as the inverse of the gradient of the depolarization times of this triangle.

\subsection{Parameter estimation and model adjustment from the experimental optical data}

Several mathematical model parameters were directly estimated from the $2 \mathrm{D}$ measurements of local conduction velocities and the duration of the action potential, specifically the anisotropy ratio $(\rho)$ and the parameter $a$. First, the conduction velocity was precisely computed from the perpendicular distance between contour lines of activation times (i.e., the axis of AP propagation). First, we determined the ratio of the velocities of depolarization wavefront measured around the tip of the stimulating electrode in the longitudinal and transverse directions (relative to the fiber

orientation). Then, the anisotropy ratio was computed using the formula $\rho=\left(C V_{\text {trans }} / C V_{\text {longit }}\right)^{2}$. 
The electrode was positioned on a relatively 'flat' area, such that the shape and curvature of the heart did not affect the calculations. Second, the other parameter, $a$, was estimated as follows: we simulated the APD90 for different values of $a$, and fit a linear function APD90 $=\mathrm{f}(a)$ to these values (as in Aliev and Panfilov 1996b); we further extracted $a$ corresponding to the mean of the APD90 values (from normal and border zones) measured at low pacing rate (when APD does not change significantly with increase in cycle length). The estimation for $a$ was performed for normal myocardium and border zones of the scar.

Finally, to evaluate the 3D model behaviour, we quantified the error in activation times between the measurements and simulations using isochronal maps of depolarization times as previously presented in the healthy heart model (Lepiller et al 2008, Pop et al, 2009a, Relan et al 2009a and 2009b). In brief, the measured isochrones of depolarization times from the optical data were mapped onto the epicardial surface and further registered with the 3D volumetric mesh of the pathological hearts. Next, we calculated the absolute error of the difference between the measured depolarization times (on the vertices where optical data is available, since only the anterior heart surface is exposed to cameras) and the simulated depolarization times. We started the adjustment with an automatic, crude initialization step which provided a global value of $d_{0}$, (for healthy ventricle); then, an iterative algorithm was used to decrease the error in the mean conduction velocity for each region. In order to obtain a regional adjustment as well as to speed up the process, the left ventricle was divided into 17 zones as per the American Heart Association (Cerqueira et al 2002). Additionally, the right ventricle was divided into 9 zones; thus we obtained a total of 26 zones where the local conductivity parameter $d_{i}$ was constant within a zone $(i)$ during the adjustment. In particular, it was previously shown (Keener and Sneyd 1998, Moreau-Villeger et al., 2006) that for planar waves we can consider the following relationship between the parameter $d$ and the speed of depolarization front (i.e., the conduction velocity, $c$ ): $\mathrm{c} \alpha(d)^{1 / 2}$. We then initialized the value of $d_{i}$ for each region from the mean conduction velocity $\overline{c_{i}}$ of that region computed from the measured depolarization times. Note that $d$ for the core of RF scar and infarct was set to zero; these were additional zones determined from MR images' segmentation using expectation-maximization method (described in the previous section). This affected the extent/geometry of some of the 26 zones containing scars but not their properties. After the initialization of the local pseudo-conductivity $d$, we iteratively improved the model fitting by employing a trust region method (Conn et al, 2000); here we implemented this using the Trilinos solver package and used an objective function to minimize the difference between simulated and 
measured depolarization times, and a volumetric $\underline{d}_{\underline{i}}$ map was obtained, for each of the 26 regions (Relan et al 2009a, 2009b). The adjustment was stopped after the mean error in activation time converged.

Lastly, we tested the sensitivity of adjusted conductivity maps to different pacing locations; for instance, we used the adjusted conductivity map obtained from one pacing location as an input to the model where we paced from another site, and compared these predictions against measurements.

\subsection{Influence of fiber orientation}

A total of 10 synthetic fibers datasets were computer generated (five synthetic fiber datasets for each heart), and the "perturbation" was implemented by allowing the helix angle to vary transmurally between the following ranges, from the endocardium to the epicardium: $-50 \%+50^{\circ}$, $60^{\circ} \%+60^{\circ},-70^{\circ} /+70^{\circ},-80 \%+80^{\circ}$ and $-90^{\circ} /+90^{\circ}$. First, the perturbation in fiber orientations can be observed qualitatively in the example below, shown for the infarcted heart: (a) fibers were extracted from DT-MRI; and (b) fibers in the synthetic datasets corresponding to various helix angles: $-50 \%+50^{\circ},-70^{\circ} / 70^{\circ}$ and $-90^{\circ} /+90^{\circ}$. Next, we computed the angular difference between the helix angle derived from synthetic fibers and the helix angle from DTI-fibers; this calculation was implemented in Matlab and the angular difference was expressed as mean \pm S.D. (over the all vertices of each mesh). Finally, we studied the influence of perturbation in fiber direction, by performing simulations with all synthetic fibers datasets and DTI fibers; this allowed us to evaluate the impact of fiber direction on activation times.

\section{Results}

\subsection{Parameter estimation from 2D optical images: anisotropy ratio and AP duration}

Two important electrophysiological characteristics were estimated directly from 2D optical images: (1) the ratio between transverse and longitudinal conduction velocities; and (2) action potential duration (APD). These estimates were used to tune the parameters $\rho$ and $a$, respectively, in the mathematical model ( $\rho$ value was computed as the squared value of the velocity ratio).

Figure 3 illustrates an example of normal wave propagation mapped from the epicardium just before the creation of an RF ablation scar. Figure 3a shows a 2D optical image of the heart 
(110x110 pixels). The spatial resolution was calculated from the calibration of the CCD camera, yielding a $0.7 \times 0.7 \mathrm{~mm}^{2}$ pixel size for this particular example. The anisotropy ratio was derived from a selected $\sim 2 \times 2 \mathrm{~cm}^{2}$ region of interest (approximately 30x30 pixels) around the tip of the stimulating electrode; this ROI is highlighted as a green box (Fig 3a). For clarity, we also present the fiber orientations determined from DT-MRI, in the same selected region (Fig 3b).

Figures $3 \mathrm{c}$ and $3 \mathrm{~d}$ illustrate the radial propagation of the impulse on the epicardium, from the pacing site. A color isochronal map of the depolarization times (after applying a 5-ms square pulse at the electrode tip) is shown in Fig 3c, with the earliest activation time in red; the first 50 $\mathrm{ms}$ are presented, as indicated in the scale. To facilitate the calculation of the anisotropy ratio, this color isochronal map is also represented as a contour map with the isochronal lines separated by 5ms intervals (Fig 3d). Near the pacing site, the fiber orientations generated concentric ellipsoidal lines of propagation; thus, we observed that the impulse conduction (here, the front of depolarization wave) is faster in the direction parallel to the fibers (long arrow) than in the perpendicular direction (short arrow). In this example, the values for measured conduction velocity in the transverse and longitudinal directions were $\sim 22 \mathrm{~cm} / \mathrm{s}$, and $60 \mathrm{~cm} / \mathrm{s}$, respectively; thus a value $1: 2.7$ for the velocity ratio was calculated (which gives an anisotropic ratio of $\rho=0.14$ ).

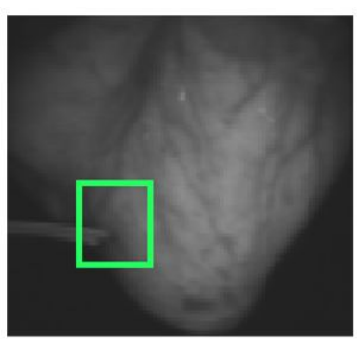

(a)

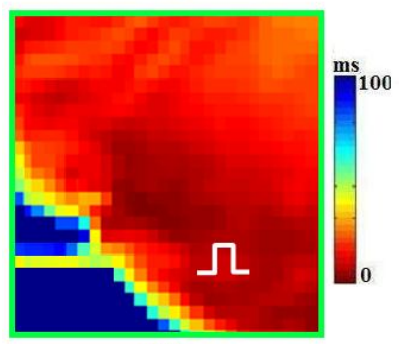

(c)

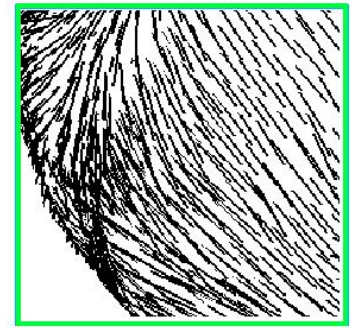

(b)

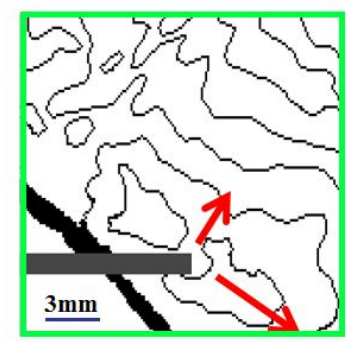

(d)

Fig. 3 Estimation of anisotropy ratio: (a) 2D optical image of a healthy heart; the box depicts the region of interest which was used for further analysis; (b) representative fibers in the ROI with their direction derived from DT-MRI; (c) color map of activation times associated with impulse conduction during the first $50 \mathrm{~ms}$ after applying a 5-ms pulse at the stimulating electrode tip; (d) isochronal contour map (lines $5 \mathrm{~ms}$ apart) used to calculate conduction velocity in the transverse (short arrow) and longitudinal (long arrow) direction, respectively, giving a velocity ratio of approximately 1:2.7 (more detail in text). 
Figures $4 \mathrm{a}$ and $4 \mathrm{~b}$ show representative examples of optical action potential waves recorded in hearts with an RF lesion and an infarct scar. We observed in detail the AP waves from normal zones and zones bordering the scar, as well as from the dense scar. It was consistently observed in the optical experiments (from all 6 hearts) that, compared to the APD in the normal zone NZ, the APD in the border zone BZ is slightly shortened in the RFA hearts, and substantially shortened in infarcted hearts. In the latter case, AP waves also had noticeably smaller amplitude, slower upstroke, and a 'triangular shape'. No optical signals (i.e., AP waves) were recorded from the core scars of the hearts, regardless of the scar type. For each type of scar ( $n=3$ hearts with RFA scar and $n=3$ hearts with MI scar), the mean APD at 90\% repolarization (APD90) was calculated for regions of interest selected from the $\mathrm{NZ}$ and $\mathrm{BZ}$, (note that the calculation of the APD in the BZ of ablation scar was to verify our hypothesis that this zone can be neglected in the model construction for the RF heart).

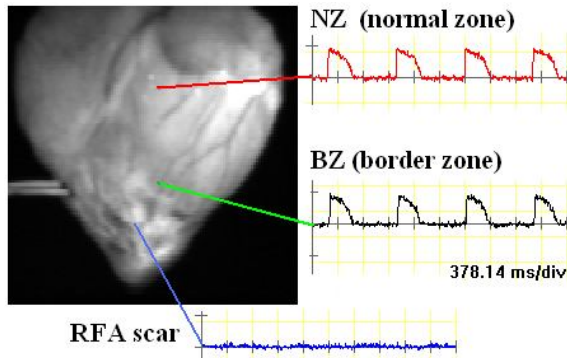

(a)

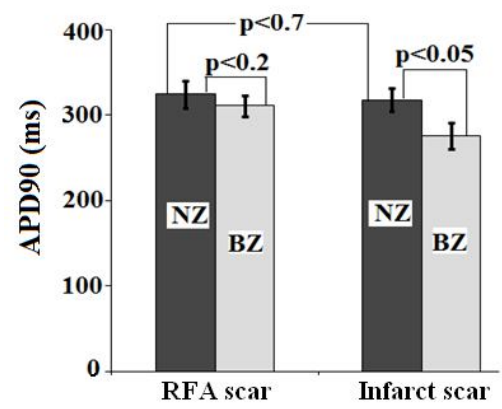

(c)

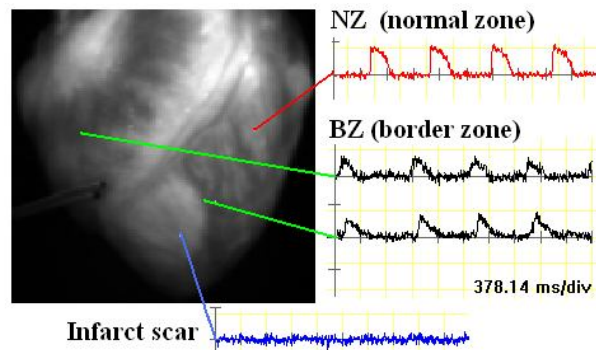

(b)

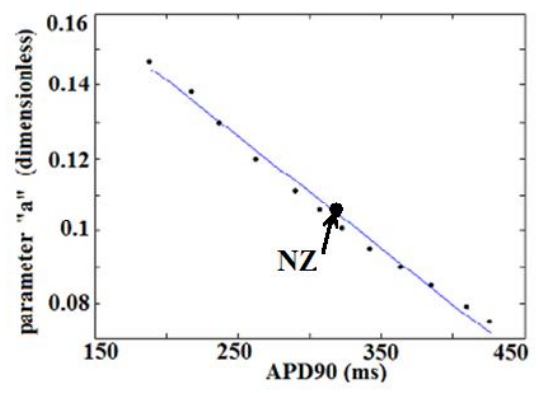

(d)

Fig. 4 (a) Model fitting for action potential duration, APD: (a) 2D optical image of a heart with an RF lesion and examples of AP waves at different pixel locations selected from the NZ, BZ and RF scar; (b) 2D optical image of a heart with a 4-week-old myocardial infarct and examples of AP waves at different pixel locations from the NZ, infarct scar and BZ (the latter has AP waves with reduced APD90 and slower up-stroke); (c) comparison between APD90 in the normal and border zones: column heights are the mean and error bars are the standard deviation (calculated from $\mathrm{n}=3$ hearts for each scar type); the one-way ANOVA analysis demonstrates a significantly smaller value for APD90 in the BZ of the infarcted hearts compared to the NZ $(\mathrm{p}<0.05)$; (d) plot of the simulated APD90 as a function of $a$ in the Aliev-Panfilov model (the small dots represent the simulated data and the line is the linear fit obtained using Matlab); example of estimation for $a$ values of 0.112 corresponding to measured APD90 in the NZ of either ablation/infarct hearts. Note that the measurements are the mean APD90 as shown in (c). 
These results are included in a bar graph and displayed in Fig 4.3c; the results of a one-way ANOVA test are also included. As expected, there was no significant difference $(\mathrm{P}=0.69)$ between the mean values for APD90 measured in the NZs of the RFA hearts $(324.6 \pm 15.7 \mathrm{~ms})$ and the NZ for infarcted hearts $(318.3 \pm 14 \mathrm{~ms})$. There was a negligible difference between the measured values in the $\mathrm{NZ}(324.6 \pm 15.7 \mathrm{~ms})$ and $\mathrm{BZ}(311.0 \pm 12.2 \mathrm{~ms})$ of the RF scars $(\mathrm{P}=0.112)$; however, in infarcted hearts, the difference in APD90 between the NZ (318.3 $\pm 14 \mathrm{~ms})$ and the BZ (275.7 \pm $15.6 \mathrm{~ms})$ was found to be significant $(\mathrm{P}<0.05)$.

Using these results, we first estimated the model parameter " $a$ " (see Fig 4d) in the NZ of the hearts as in (Aliev and Panfilov, 1996b), for $k=8$. We plotted several computed APD90 data points obtained in the simulations by varying the " $a$ " value in the computer model. The plot yielded in a first approximation a linear fit (using Matlab) and allowed us to find the corresponding parameter " $a$ " for the mean APD90 values measured in the NZ of the infarct and ablated hearts $(\mathrm{a}=0.112)$. Note that due to the small difference in the APD90, we consider the parameter " $a$ " to have similar values in the NZ and BZ of the hearts with RF ablated scars.

In addition, after performing simulations with various values for $k$, we found that $k=8$ reproduces correctly the AP wave up-stroke obtained in optical experiments; thus, this value is also a good match for the porcine healthy tissue. However, in order to derive $k$ for the border zone BZ, we used a simplistic approach (i.e., 'trial and error') and derived a generic pair of values $(a, k)$ that reproduced several characteristics of the AP wave. This pair was find to be the best match to the average measurements for the upstroke derived on a ROI selected from the BZ of the infarct, having set a conductivity three times smaller than in the healthy tissue. Our analysis yielded the following pair of values for BZ: $a=0.2$ and $k=2$; this values could be generically used in the Aliev-Panfilov model, to simulate the characteristics of the AP wave in the ischemic BZ for hearts with 4-5week old infarction. 


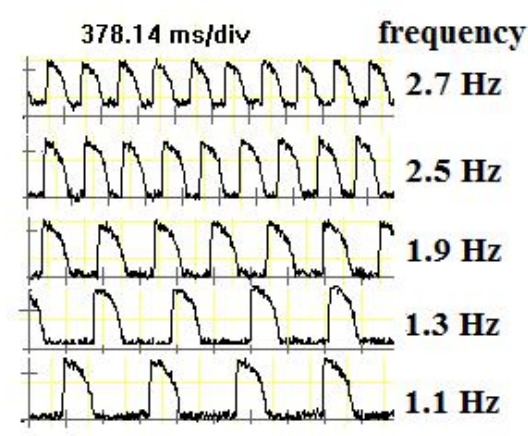

(a)

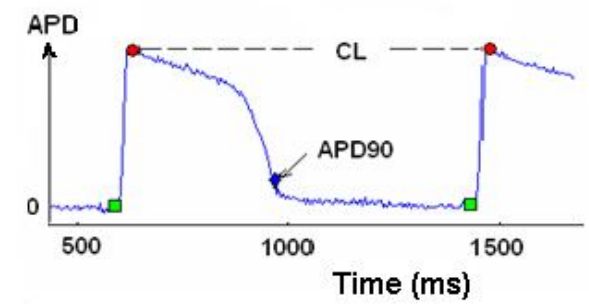

(b)

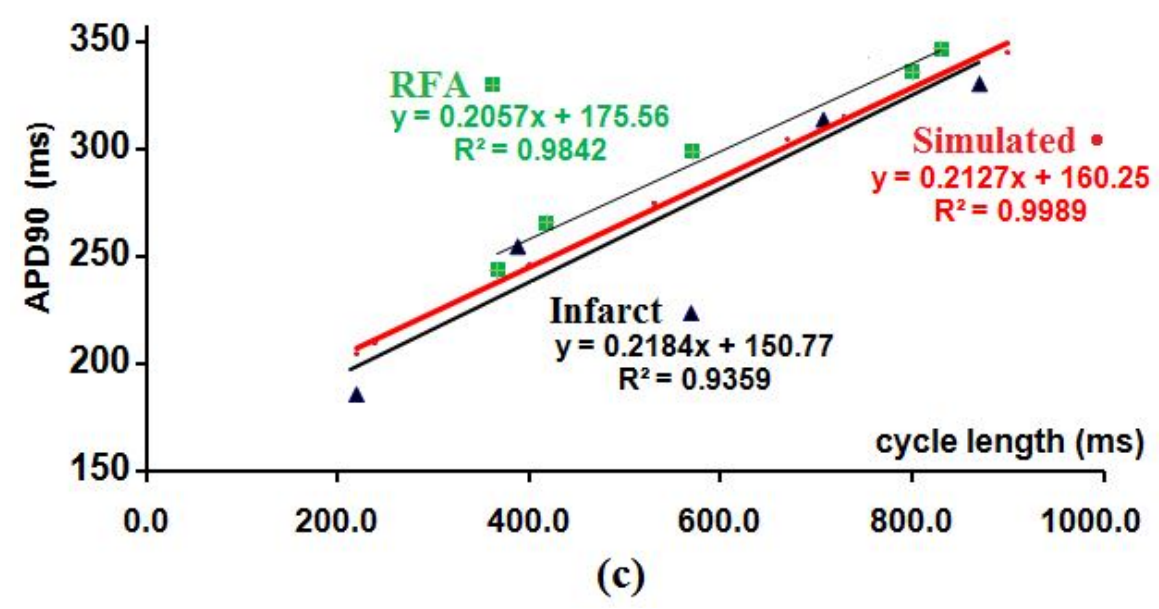

Fig. 5 Experimental and simulated restitution curves for APD90: (a) representative AP waves measured at the same pixel location obtained in the heart with an RF scar, at different pacing frequencies; (b) example calculation of the APD at $90 \%$ repolarization for a cycle length $\mathrm{CL}=830 \mathrm{~ms}$; and (c) measured restitution curves (the square dots correspond to the data points measured in the RF scar heart, the triangle dots represent the data points for the infarct heart and the circle dots are the simulated data points (for the simulated curve).

Figure 5 demonstrates the results obtained for experimental and simulated restitution curves of APD90. Figure 5a shows representative optical AP waves recorded from a NZ of the heart with an RF scar (presented in Fig 5a) obtained after stimulation at different five frequencies (these waves are illustrated using the BV-Ana software). As expected, the APD shortened as we increased the pacing frequency (which is equal to the inverse of the cycle length, CL). Similar behaviour was observed in a NZ of the infarcted heart stimulated at four different frequencies. For the actual calculation of APD at each frequency, we determined the average APD90 (APD at 90\% recovery time) on selected regions from the normal zone of the left ventricles, comprising AP waves from at least 200 pixels (see Fig 5b). These measured restitution curves APD = f (CL) are 
plotted in Fig. 5c and compared to a simulated curve for which the average parameter $a=0.112$ (found above for the NZ) was used as an input to the mathematical model. A good correspondence between the slope of the experimental restitution curves and the simulated curve was observed; thus, it appears reasonable to use the simulated curve for healthy tissue in computer models built for porcine hearts.

\subsection{Construction of 3D MRI-based models and adjustments of conductivity maps}

Figure 6a shows long- and short-axis MRI sections of the heart with an RF scar using an FSE sequence (a week after preservation in $10 \%$ formalin). The ablated lesion showed a reduction of MR signal intensity; this is due to water dessication, a characteristic specific to coagulative necrosis processes following severe thermal damage. A very thin, well demarcated border (yellow arrows, in Fig 6a) can be visualized between the scar and the surrounding normal myocardium.

A whole-mount histopathology slice stained with Masson's Trichrome and scanned at $10 \mu \mathrm{m}$ resolution is presented in Fig $6 \mathrm{~b}$. Overall, we demonstrated very good correspondence in the shape and extent of this acute RF lesion between histology and MRI. Specifically, in the selected regions (shown in higher magnification in Fig 6b), we observed normal tissue with healthy myocytes and preserved architecture, whereas the ablated scar has typical characteristics of acute thermal damage (with ruptured, dead myocytes and severely disorganized bundles). Notable in histology is a very thin rim, as observed in MRI; this rim is likely due to accumulation of edematous fluid and some red blood cells at the BZ. The myocytes are viable in the BZ; thus, most likely this rim is responsible for the slightly reduced APDs observed in the optical recordings from these areas. Our initial hypothesis was that this rim (border zone) can be neglected and the APD measures confirm this; thus, we proceed further with the construction of a 3D MRI-based model with two zones (ablation scar and healthy zone). 


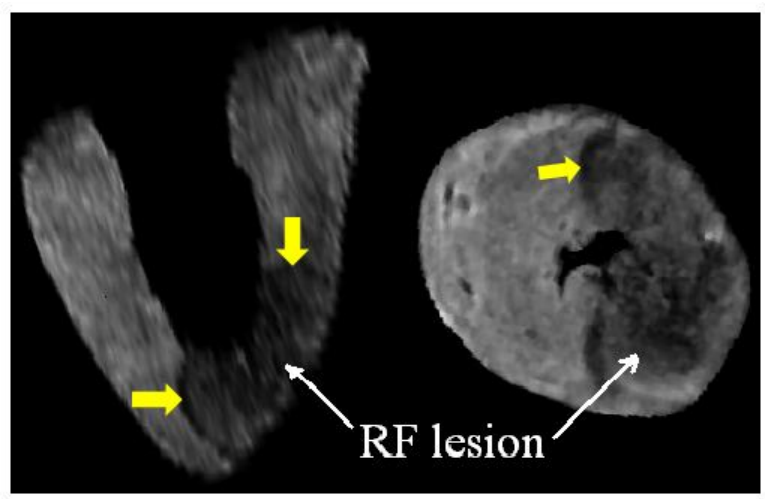

(a)

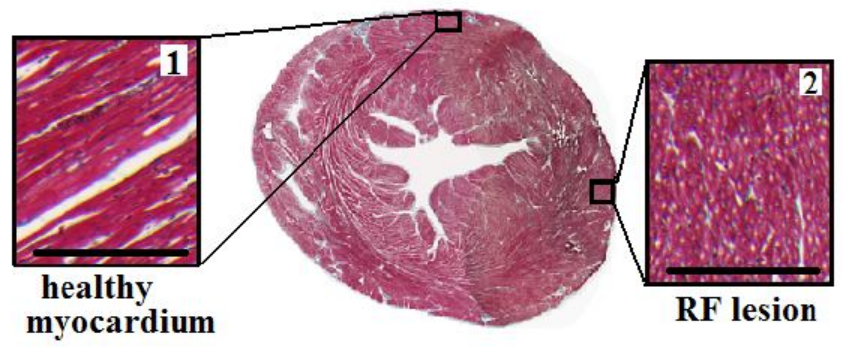

(b)

Fig. 6 Characteristics of the heart with RFA scar observed in MRI and histology: (a) MR images in longitudinal and short-axis views; yellow arrows point to the border between healthy myocardium and the dense scar (white arrows); and (b) Masson's Trichrome stain of a whole-mount histology sample corresponding to the slice presented in the MRI short-axis view, with selected zoomed-in insets from the healthy and ablated areas, respectively, demonstrating loss of architecture and viability in the RFA lesion (scale bars: $300 \mu \mathrm{m}$ ).

Figure 7 shows the resulting 3D MR image-based computer model constructed for the heart with an RF ablation scar. The 3D computational volumetric mesh (of 180,000 tetrahedral elements) was constructed from anatomical MR images. However, based on the optical and MRI observations, this 3D model has only two segmented zones: normal myocardium and RF scar (Figure 7a). Figure $7 \mathrm{~b}$ illustrates the fiber directions as specified at each vertex in the mesh. The mesh segmentation corresponding to the 26 AHA zones is shown in Fig. 7c (three zones are highlighted). 


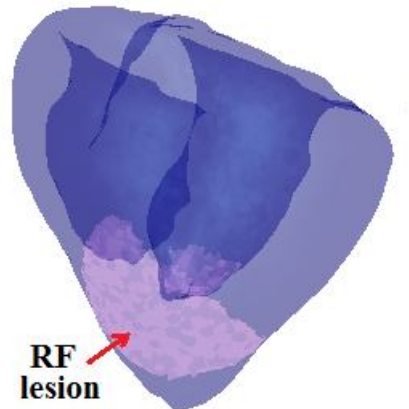

(a)

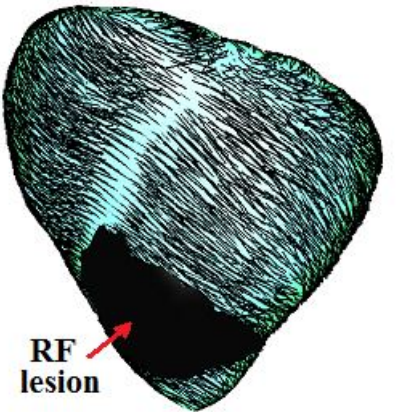

(b)

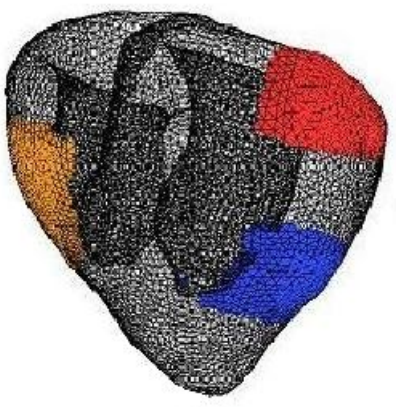

(c)

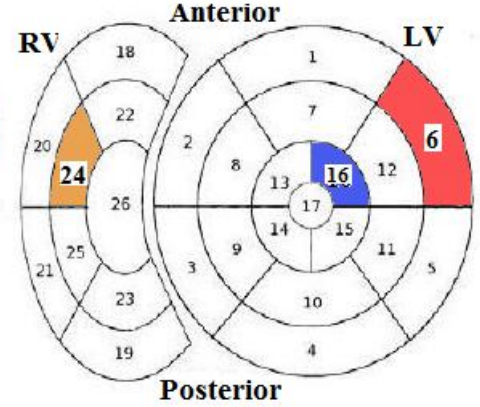

(d)

Fig. 7 3D MRI-based model construction for the heart with RF scar: (a) 3D volumetric model with two segmented zones (normal myocardium and RF scar); (b) fiber directions integrated onto the model; (c) the tetrahedral mesh and three highlighted AHA zones out of a total 26 AHA zones presented all in a planimetric view in $(\mathrm{d})$.

The volumetric adjustment of conductivity maps is based on the comparison between experimental and simulated isochronal maps, and is schematically presented in Figure 7. The model is initialized with literature values for global conductivity with all AHA zones having the same values $\left(\mathrm{d}_{0}\right)$. As mentioned in section $\mathbf{2 . 3}$, in the $3 \mathrm{D}$ model, the scar is assigned a zero value for conductivity. The other two model parameters ( $a$ and $\rho$ ) were globally tuned in the model using the values obtained in the section 3.1. 


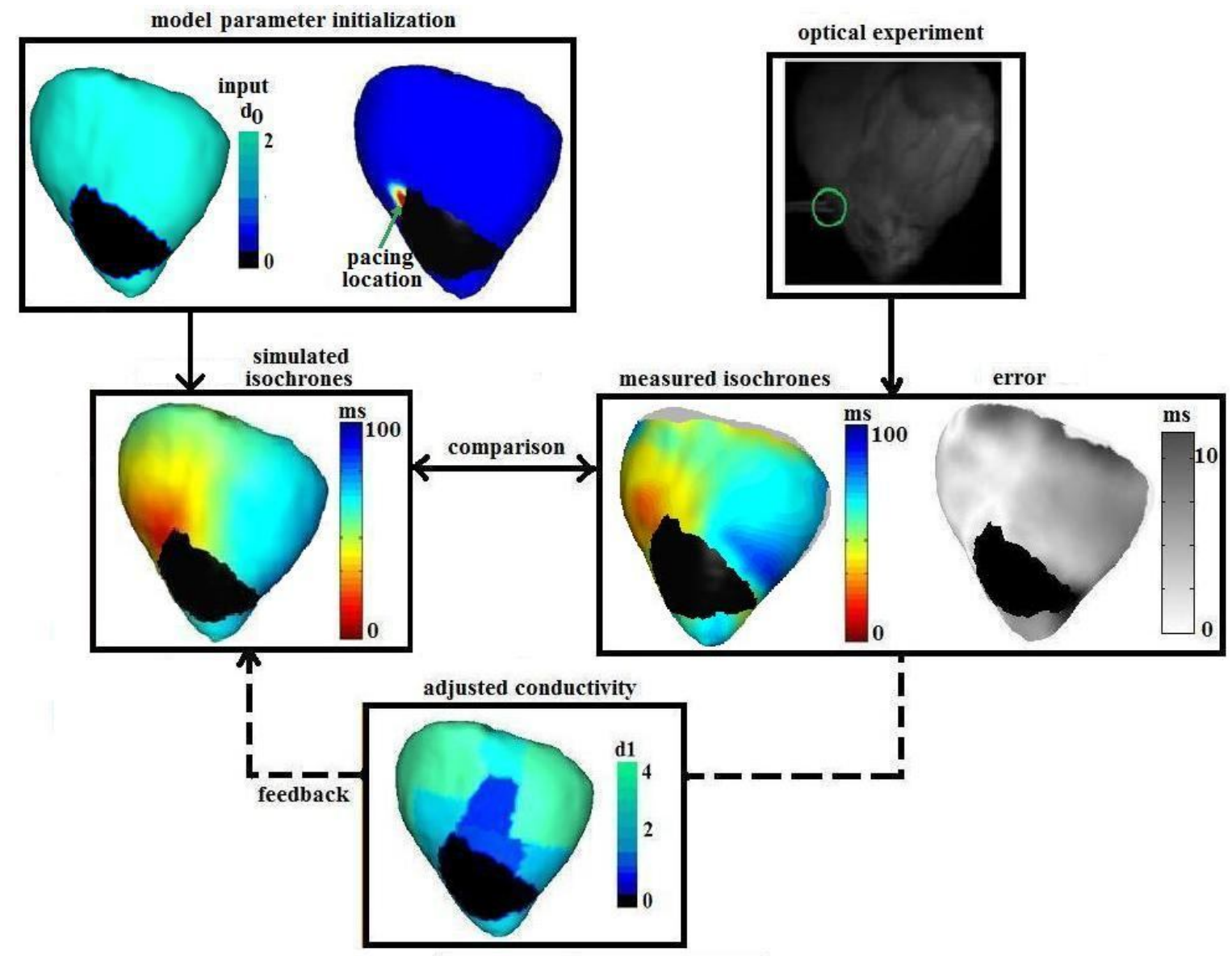

Fig. 8 Example of a 3D volumetric adjustment of conductivity $d$ in the heart with RF scar; with model initialization (d0-map and pacing location) and final results (d1-map) and associated mean error in activation times after adjustment.

The pacing site in the simulation corresponds precisely to the electrode tip location in the optical recordings and has the earliest activation time in both the simulated and experimental color isochronal maps, where the associated scales are in $\mathrm{ms}$ (red is $0 \mathrm{~ms}$ and corresponds to the earliest activation time; blue corresponds to late activation time with $120 \mathrm{~ms}$ being the latest time). The average error between the simulated and experimental depolarization times was iteratively reduced from $57 \mathrm{~ms}$ to $10 \mathrm{~ms}$ (that is approximately 6-fold); the adjusted 3D conductivity map (d1) is included in Figure 8. We observed a slower conductivity within the LAD territory (most likely due to some fat deposition around the vessel) as well as around the RF scar, which was expected because anatomical blocks are known to reduce the conduction velocities in their proximity. 
The adjustment process involved re-evaluation of the local conductivity $d_{i}$ in each zone; a histogram of the conduction velocities computed on the epicardium (at each vertex in the mesh) using the d1-map after the last adjustment step is presented in Figure 9a. The histogram of velocities computed from the optical isochronal map is shown in Fig 9b. An absolute error between simulated and experimental conduction velocity is shown in Fig 9c.

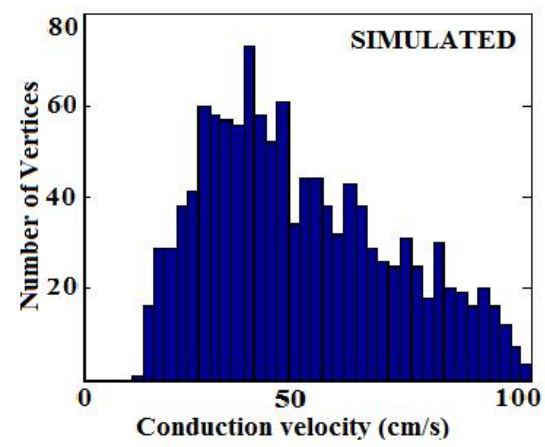

(a)

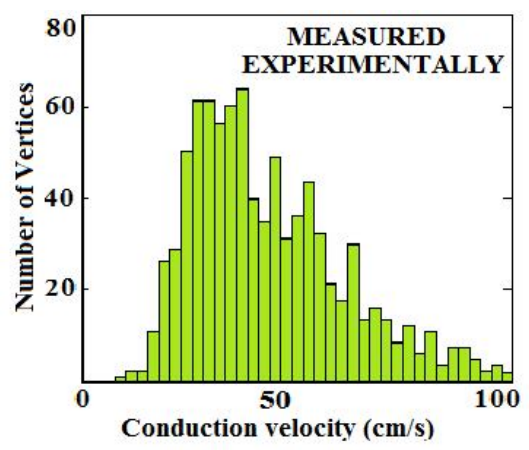

(b)

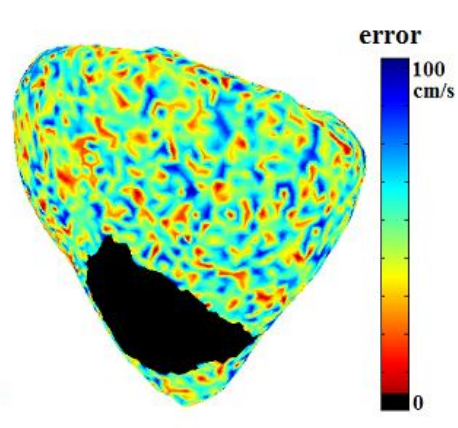

(c)

Fig. 9 Histogram of computed conduction velocities (at each vertex) from: (a) simulated isochrones after adjustment; (b) experimentally measured isochrones; and (c) corresponding absolute error between conduction velocity maps calculated at each vertex and interpolated on the 3D mesh.

The error in conductivity maps and sensitivity to different pacing location is presented in Figure 10. The second pacing location is shown in Figure 10a; the corresponding volumetric dmap for the second location is shown in Figure 10b. The absolute difference (d1-d2) between the adjusted d-maps (from the two pacing locations) is shown in Figure 10c. Evolution of error in the simulated depolarization times during the adjustment (first 20 iterations) is illustrated in Fig. 10d. Furthermore, the sensitivity of model prediction to pacing location was tested by computing the isochronal maps at the second pacing location and using $\mathrm{d} 1$ as input for conductivity values; the error between the measured isochronal maps and these simulated isochrones is presented in Fig 10e. The Fig 10f shows the correlation plot between computed conductivities from one pacing location (D1) vs. the values obtained per zone from the other pacing location (D2), yielding a coefficient $r=0.89$ for this RF heart. The Bland-Altman analysis (Fig 10g) demonstrated no significant bias between these values, thus any of the maps can be used as input to the specific model, for each heart. 


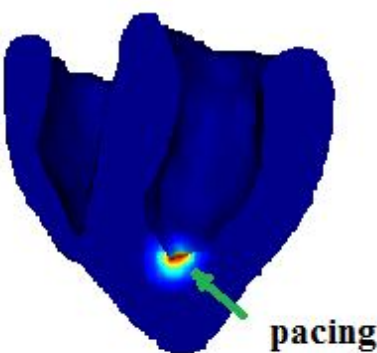

(a)

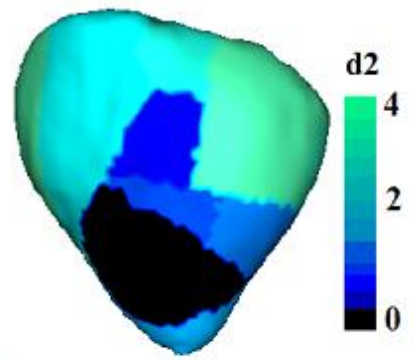

(b)

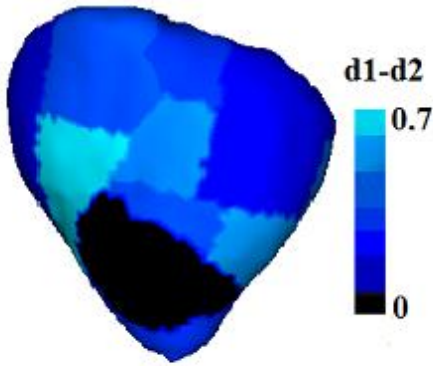

(c)

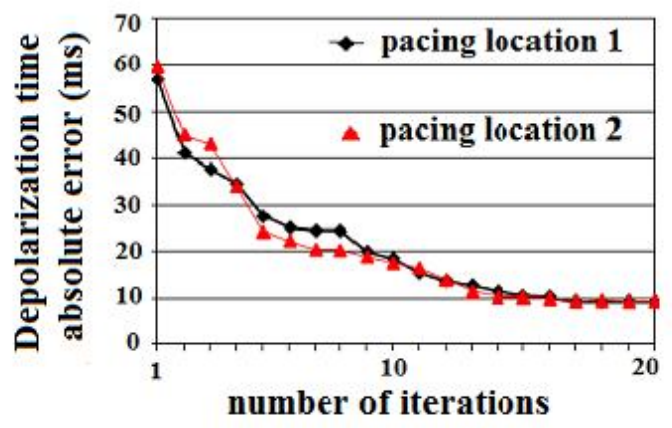

(d)

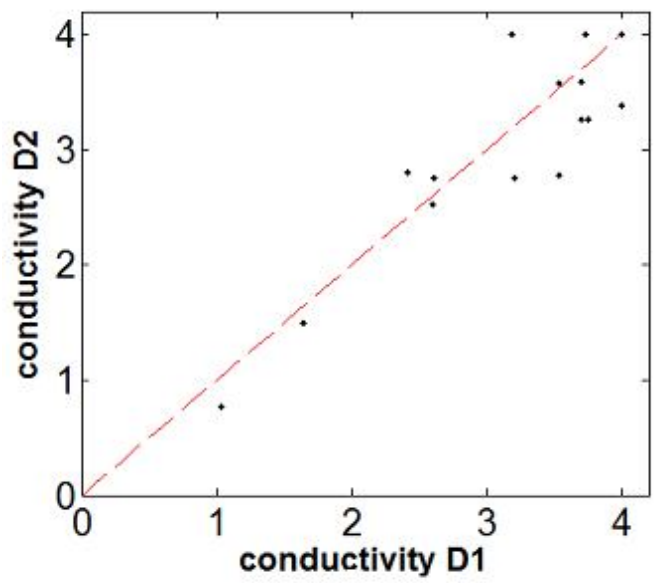

(f)

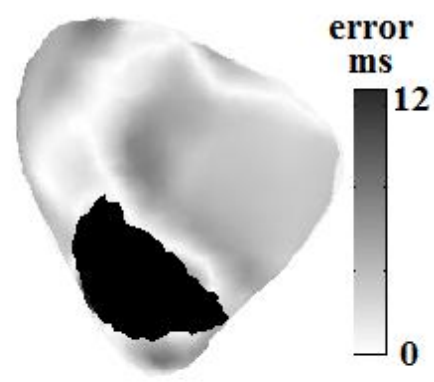

(e)

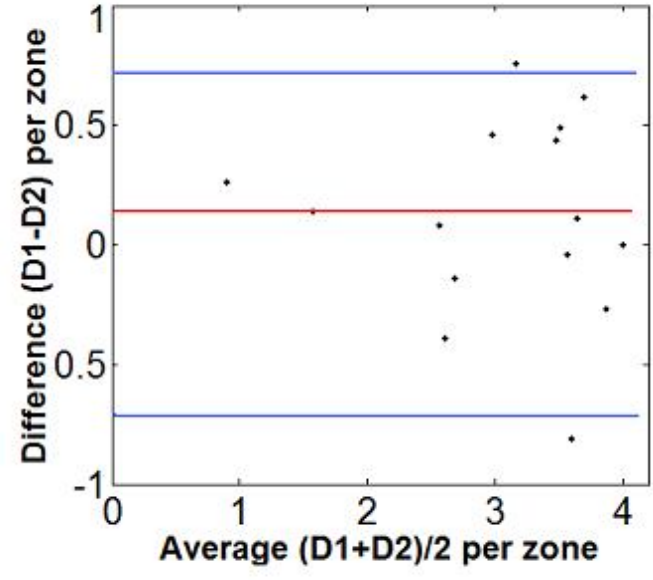

(g)

Fig. 10 Volumetric adjustment of conductivity for the second pacing location indicated in (a); (b) its computed d-map; (c) absolute error between d-maps at the two locations; (d) evolution of error in activation time during the iterative adjustment, (e) sensitivity of model prediction to pacing location shown by the mean error in depolarization time, (f) correlation plot between the conductivity values derived by zone for different pacing conditions, yielding a correlation coefficient of 0.89 (for the RF heart), and (g) associated Bland-Altman analysis, with the red line corresponding to the mean difference and the blue lines correspond to \pm 2 S.D. (note that some zones have the same value for $d$, see text for detail). 
The following results were obtained from heart with a chronic infarct scar (4 weeks old). The construction of the 3D MRI-based computer model has a similar pipeline as the one presented above.

Figure 11 shows a 3D view through the MRI volumetric data set; here we present a 3D volumetric map of the ADC (ADC) used for the construction of the model. Some heterogeneity in MRI signal intensity was observed (see figure 11a); the bright areas correspond to an expected increase in ADC values in the infarct scar areas. The fiber directions derived from DT-MRI (fig 11b) are disturbed in the infarct area; we observed a severe disarray of the bundles and significant deviation from the normal orientation. The large infarct area (in the LAD territory) was observable during gross visual inspection after the heart was preserved in formalin (Fig 11c) and also corresponded well with the scar extent observed in the optical images (Fig 11b). The infarction involved both right and left ventricles (note: the markers were used to register the optical images with the mesh derived from MR images).

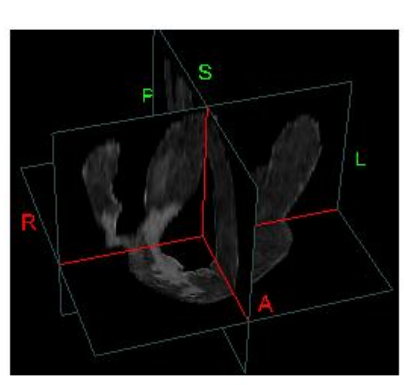

(a)

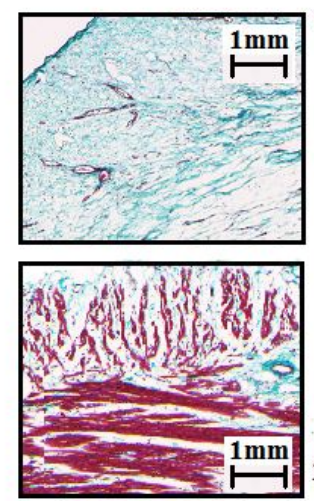

1. scar
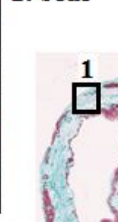

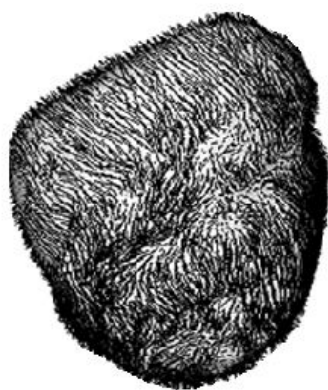

(b)

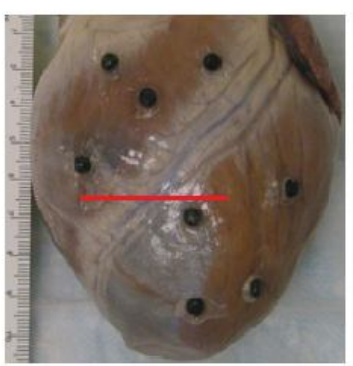

(c)
3. healthy

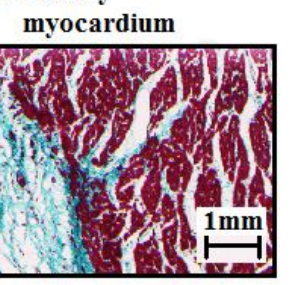

(d)

Fig. 11 MRI and histology of the infarcted heart: (a) reconstructed 3D ADC volume from MR scans, with the infarct delineated by the bright areas; (b) reconstructed fiber directions from DTMR images; (c) gross inspection of the ex vivo heart after preservation in formalin - the horizontal red line corresponds to the height from which the cross-section slice was taken for whole-mount histology; and (d) Masson's Trichrome specifically stains healthy myocardium in red and collagen in blue; selected areas from the NZ, BZ and dense scar are shown at higher magnification. 
Further analysis using histological whole-mount preparation of a slice stained with Masson's Trichrome is shown in Fig 11d; the slice was taken at the height indicated by the red line in Fig 11c. On microscopic observation it appeared that infarct areas remodeled over time. The core scar (stained blue) has large areas with myocytes replaced by dense, collagenous fibrosis, which is also accompanied by a complete loss of anisotropy. The border zone areas (border zone, BZ) have mixed islands of viable and non-viable myocytes, as well as fiber directions slightly changed due to droplets of collagen deposits between surviving cardiac myocytes (viable myocytes stained red). Most importantly, the selected areas shown with higher magnification in Fig 11d (NZ, BZ and scar) also showed differences in optical signals recorded from these zones, with no signal (AP waves) in the scar as well as altered morphology of AP waves in the BZ.

Results yielding to the construction of 3D MRI-based model in the infarcted heart are shown in Figure 12. First, the classification results obtained using the EM algorithm, are presented in figures 12 (a-b), where the histogram in (12a) clearly illustrates the mixture of the Gaussian distributions mixture whereas in (12b) we illustrate the Gaussian distribution for NZ and scar (note that the distribution for BZ does not necessarily have to be Gaussian). Next, after tetrahedrization, a resulting mesh (see Fig12.c) of $\sim 270,000$ elements and with fiber directions specified at each node was obtained; this mesh has three morphological zones: NZ, BZ and scar, (see red areas corresponding to BZ and green areas corresponding to scar in Fig. 12e); dense scar had zero electrical conductivity. Presented in Fig 12d is a short-axis slide from the 3D ADC map, and its corresponding segmentation into the three zones (Fig 12e). A good histological correspondence was found for these three segmented areas (see Fig 12f). 


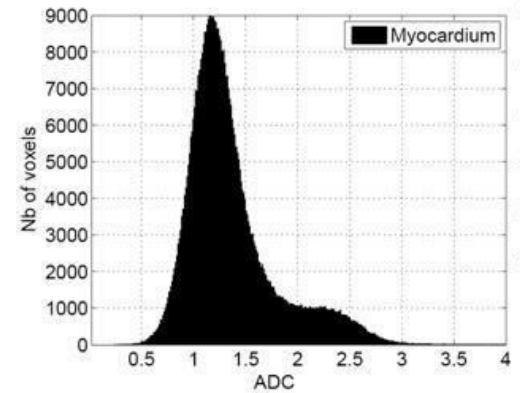

(a)

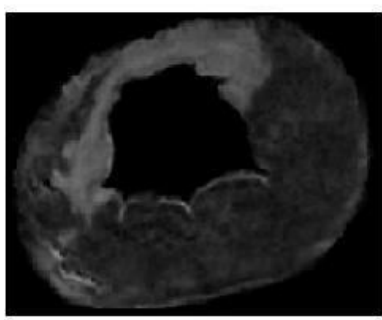

(d)

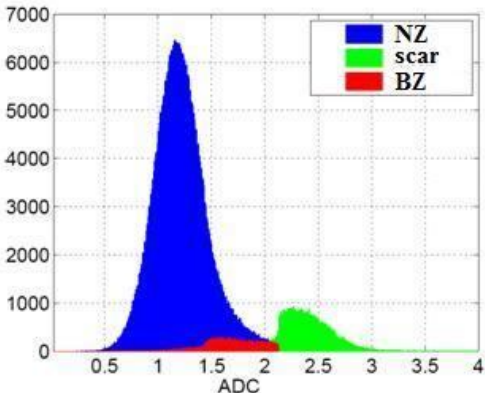

(b)

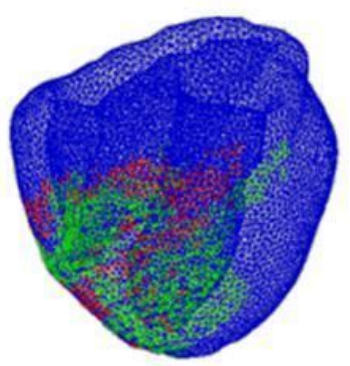

(c)

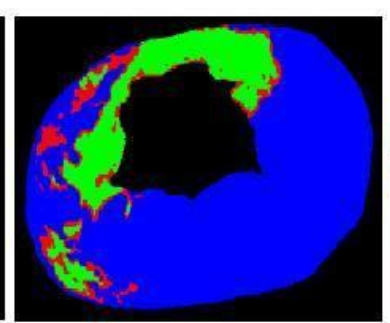

(e)

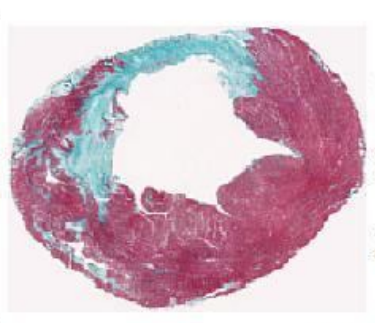

(f)
1. NZ

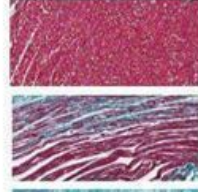

3. scar

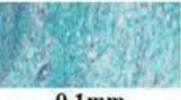

$0.1 \mathrm{~mm}$

Fig. 12 Results from the model construction of infarct heart: (a) histogram showing a Gaussian mixture distribution for the myocardium; (b) histogram of each zone; (c) constructed 3D volumetric mesh with the infarct zone highlighted in green; (d) a short-axis ADC map from DW-MRI, and resulting segmentation into three zones); and (e) corresponding Masson's Trichrome histology with magnified areas from the healthy/normal myocardium, border zone and the dense scar. Note that in (b), (c) and (e) NZ is blue, scar is green and $\mathrm{BZ}$ is red.

After the adjustment of the local conductivity, the experimental isochronal color maps (projected onto the 3D mesh) as well as simulated isochronal maps from the one pacing location are presented in Figure 13, where red represents the early activation time $(0 \mathrm{~ms})$ and blue corresponds to the latest activation time (here 150ms). For values of $\rho$ and $a$ in NZ and BZ we used the parameters derived in Section 3.1. Good qualitative agreement between the depolarization times can be observed from the color isochronal maps (Fig 13b and 13d). Moreover, the absolute error in depolarization time (after the adjustment) is presented in Fig 13e. 


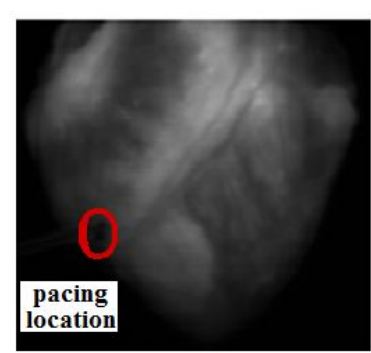

(a)

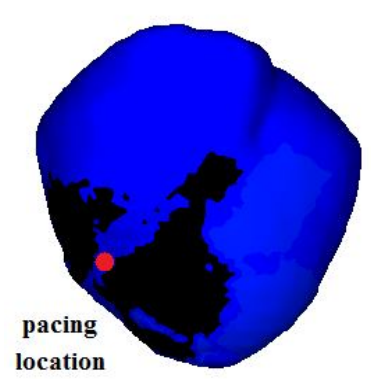

(c)

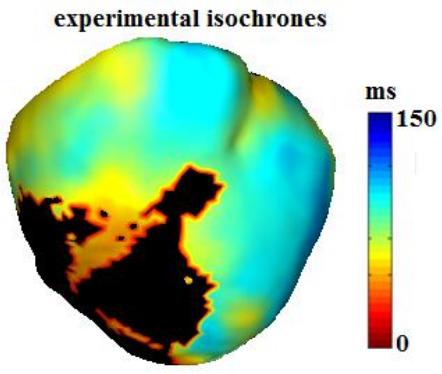

(b)

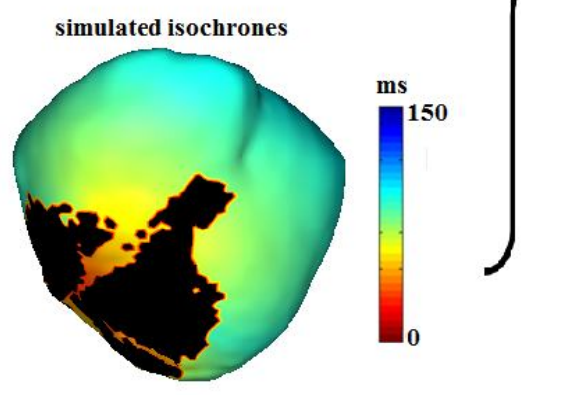

(d)

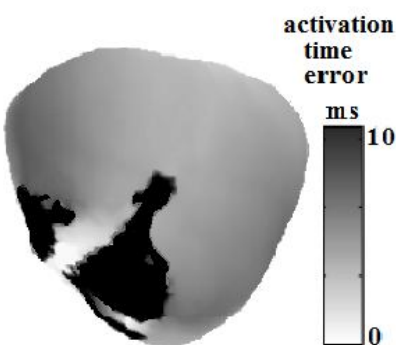

(e)

Fig. 13 Comparison between experimental and simulated isochrones (after adjustment) in the infarcted heart: (a) 2D optical image showing the position of the electrode; (b measured isochronal map computed for depolarization times; (c) the 3D model in blue corresponding to resting phase, just prior the application of the stimulating pulse (location indicated by the red bullet); (d) simulated depolarization times; and (e) corresponding error (i.e., absolute difference in activation time) after adjusting the local conductivity.

Figures 14a-b show the resulting conductivity maps after the adjustments were performed for two stimulation locations. The areas of relatively low local conductivity (i.e., associated with slow conduction velocity) were found to be around the infarct (in the BZ) and around the large vessel; the corresponding absolute error for $d$ is plotted in (Fig 14c). Figure 14d illustrates the evolution of mean absolute error in activation times (i.e., difference between measured and simulated depolarization times) calculated at each iteration during the adjustment. For the second pacing location, the depolarization time error converged to a minimum value (from $56 \mathrm{~ms}$ to $8 \mathrm{~ms}$ ) after approximately 30 iterations (requiring 12 hours of computation time); however, note that the error in activation time is already reduced to approximately $10 \mathrm{~ms}$ after 8 iterations. Fig 14e shows the correlation plot between computed conductivities from one pacing location (D1) vs. the values obtained per zone from the other pacing location (D2), yielding a coefficient $r=0.77$ for this infarcted heart. The Bland-Altman analysis (Fig 14f) demonstrated no significant bias between these values, thus any of the maps can be used as input to the specific model, for each heart. 


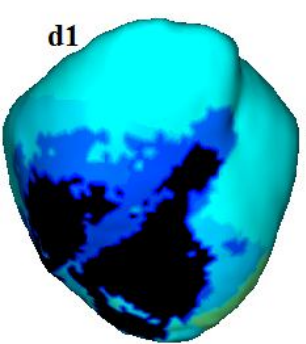

(a)

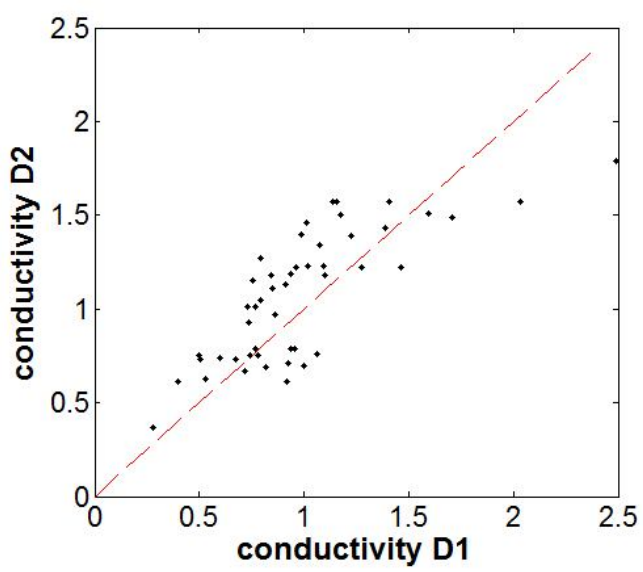

(e)

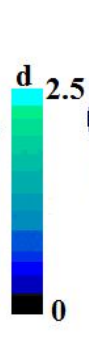

(b)

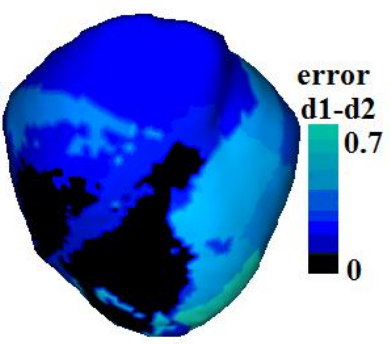

(c)

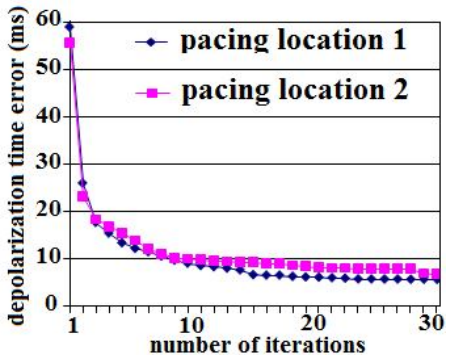

(d)

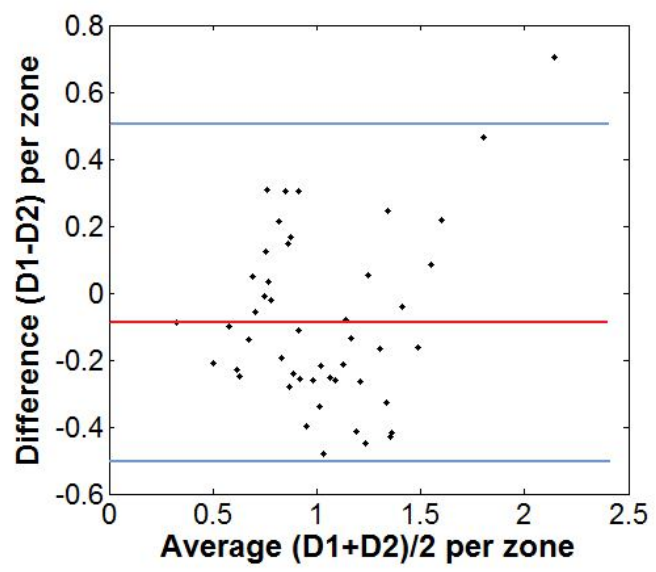

(f)

Fig. 14 Volumetric adjustment of the conductivity maps in the infract heart: (a) and (b) are resulting $3 \mathrm{D}$ conductivity maps derived from adjustments associated with two different pacing locations; (c) the corresponding absolute difference between the d-maps obtained from 2 pacing locations (note: all scales have normalized $d$ ); (d) evolution of the mean absolute error in depolarization time as it converged to a minimum during the iterative adjustment process; (e) correlation plot between the conductivity values derived by zone for different pacing conditions, yielding a correlation coefficient of 0.77 (for the infarcted heart), and (f) associated BlandAltman analysis, with the red line corresponding to the mean difference and the blue lines correspond to \pm 2 S.D. (note that some zones have the same value for local $d$ )

Overall, our results demonstrated that a simple mathematical model of electrophysiology (Aliev-Panfilov model) can be customized using the fusion of data from optical and MR imaging. Several parameters can be directly estimated from 2D optical action potential measurements; in addition 3D volumetric adjustment of local conductivity is feasible for both models built from pathologic hearts (i.e., with either RF scar or infarct scar), with resulting conductivity maps being relatively independent of pacing location.

Regarding the impact of perturbation in fibers orientation, among the resulting datasets, we found that the smallest difference in helix angles relative to the data with fiber orientation estimated from DTI, corresponded to the synthetic data with a helix angle varying transmurally 
from $-70^{\circ} / 70^{\circ}$. Below are the associated histograms for angular differences for this dataset, which are now included in the revised manuscript as: Figure 15a and 15b, respectively.

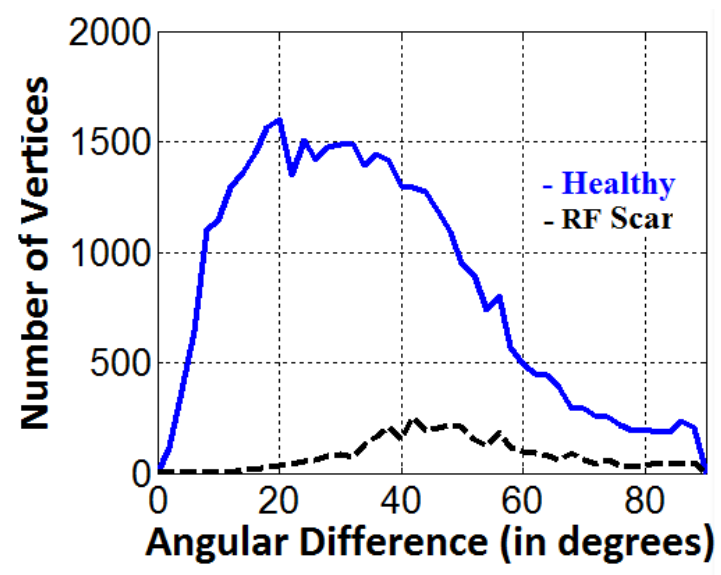

(a)

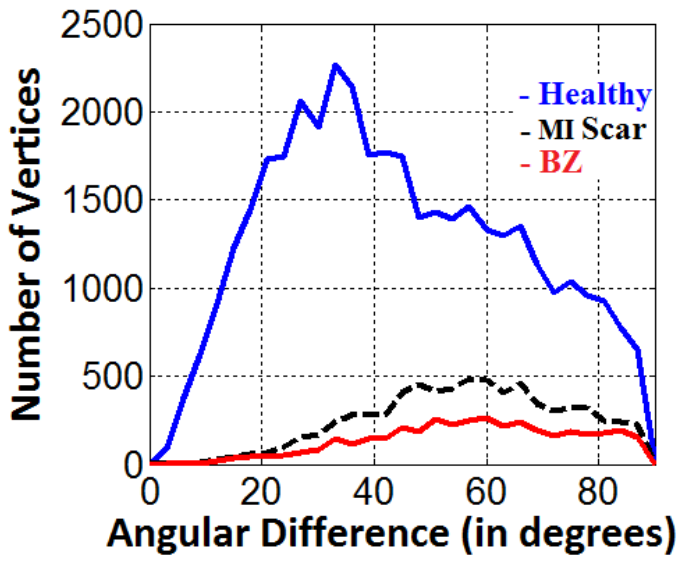

(b)

Figure 15 Histograms for angular differences between DTI and synthetic fibers, with orientations ranging over $-70 /+70^{\circ}$ : (a) RF heart and (b) infarcted heart.

Specifically, for the RF scar we obtained a helix angular difference (between fibers from DTI and synthetic fibers) of $37.8 \pm 22.4^{\circ}$ in the healthy area and $48.4 \pm 20.8^{\circ}$ in the RF scar (lesion). For the scar with myocardial infarct MI, we obtained a helix angular difference of $44.3 \pm 23.2^{\circ}$ in the healthy area, $51.3 \pm 22.2^{\circ}$ in the $\mathrm{BZ}$ and $60.3 \pm 21.7^{\circ}$ in the dense scar.

Simulations were also performed using all synthetic fibers datasets, for both pacing location on each heart (i.e., a total of 20 new simulations). Then, the computed activation times were compared with the ones obtained using the fiber directions from DTI (note that these simulations were performed with adjusted conductivity maps). To quantify the differences between the simulated action potentials with the DTI fibers and with synthetic datasets, we calculated (for depolarization times at all vertices in the mesh) the R.M.S. error and the Pearson's correlation coefficient $(r)$ together with the associated mean \pm S.D $(\mathrm{ms})$. All associated results are presented in Table 1 (see Supplemental Material A). This error analysis was performed by implementing in Matlab the equations below, which are similar to those given in (Muzicant and Henriquez, 1998):

$$
r=\frac{N \sum A T_{\text {simDTI }} A T_{\text {simSynth }}-\left(\sum A T_{\text {simDTI }}\right)\left(\sum A T_{\text {simSynth }}\right)}{\sqrt{\left(N \sum A T_{\text {simDTI }}^{2}-\left(\sum A T_{\text {simDTI }}\right)^{2}\right)\left(N \sum A T_{\text {simSynth }}^{2}-\left(\sum A T_{\text {simSynth }}\right)^{2}\right)}}
$$




$$
R M S_{-} \text {error }=100 \times \sqrt{\frac{\sum\left(A T_{\text {simDTI }}-A T_{\text {simSynth }}\right)^{2}}{\sum\left(A T_{\text {simDTI }}\right)^{2}}}
$$

where $\mathrm{N}$ is the number of vertices in the heart mesh. $\mathrm{AT}_{\text {simDTI }}$ and $\mathrm{AT}_{\text {simSynth }}$ are the simulated activation times (i.e., depolarization times, in ms) using DTI fibers and synthetic fiber data, respectively.

From the analysis of these activation times, the datasets with helix angles varying from $+/$ $60^{\circ},+/-70^{\circ}$ and $+/-80^{\circ}$ have a small RMS error, small associated difference in activation times (mean \pm S.D), and very good correlation coefficients. For the heart with RF lesion, the epicardial pacing produced better results (compared to the endocardial LV pacing location \#2), whereas for the infarcted heart, the pacing location \#1 (on the BZ, between the scars) resulted in larger error, suggesting that the epicardial pacing location \#2 (i.e., on the RV and slightly away from low conductivity zones) gives better results. However, in both cases, we concluded that the synthetic fibers can be used within an acceptable margin of error for the activation times, when DTI data is not available.

Finally, to evaluate the impact of global vs. local parameterization for conductivity parameter, another sub-set of simulations was additionally performed using global parameterization (i.e., constant values on all zones corresponding to a certain categorization) for conductivity, that is, $d$ having values of 1,2 and 3 respectively. We then compared the activation times obtained using this global $d$ parameterization and the locally adjusted conductivity maps. The difference between these computed activation times, was expressed in terms of RMS error, and mean $\pm \mathrm{SD}$ (ms). For instance for the infarcted heart, using $d=1$ in the healthy zones, RMS was $46.7 \%$, and mean $37 \pm 16.9 \mathrm{~ms}$; for $d=2$, RMS error was $15.9 \%$ and mean $9.4 \pm 4.9 \mathrm{~ms}$; and for $d=3$, RMS error was $21.3 \%$ and mean $11.2 \pm 5.7 \mathrm{~ms}$. From this analysis, we concluded that global values of $d=2$ (and $d=3$ ) could be used for the conductivity parameter of the healthy tissue for hearts 7-8 cm in height and meshes having 1-1.2mm element length (since $d$ is scalable as a function of computational domain size and elements size as well), with an acceptable error. 


\section{Discussion}

The development of 3D image-based cardiac electrophysiology models is gaining considerable attention since they can provide insights into the transmural propagation of electrical waves through the heart, complementing surfacic measurements (Chinchapatnam et al 2008) and into the causes of arrhythmogenesis (Vadakkumpadan et al 2010). In particular, parameter estimation and further customization of the 3D MR image-based cardiac models is now regarded as a very important step in this emerging cardiac field. However, progress is currently hampered by either the lack of experimental electrophysiological data obtained particularly in large, pathologic hearts or by the number of parameters to be estimated. The main 3D MRI-based computer model ingredients are: 1) a computational mesh built from anatomy and structural imaging scans (with fiber directions integrated to realistically simulate anisotropic wave propagation); and 2) mathematical equations, modelling the propagation of impulse conduction (often, the models' output is the action potential, AP). Multi-scale integrative models are highly demanding in terms of computational time, but most importantly, they contain complex details at different spatiotemporal scales. Several input parameters though are fundamental, and are present in most of the cardiac models. These include the anisotropy ratio between transverse and longitudinal conduction velocities forms the diffusivity tensor $(\tilde{D})$. Model customization consists in tuning the input parameters such that the main output (i.e., the AP wave and its characteristics of duration, shape, and upstroke) is close to measured behaviour.

In this work, we specifically characterized the propagation of AP in pathologic hearts using optical fluorescence imaging and a 3D MR-image-based computer model that uses a simplistic monodomain approach based on a macroscopic formalism combined with reaction-diffusion equations (i.e., the Aliev-Panfilov model). Several contributions are novel to the field including, to the best of our knowledge, the following: 1) the mapping of optical fluorescence AP in large pathologic hearts; 2) a 3D MR-image-based model of porcine hearts integrating anatomy, fiber directions and myocardial tissue heterogeneities (healthy vs. scar and border zone) with histological validation; and 3) model customization by estimating several parameters from 2D optical images (anisotropy ratio, duration of AP), as well as volumetric adjustment of local conductivity from maps of depolarization isochrones.

It is the choice of the researcher which computer model to use; such selection is always based on the purpose of the study (Clayton and Panfilov, 2008). For instance, one can use the Eikonal model computes only the propagation of the depolarization wavefront (Keener and 
Sneeid, 1998); this model is fast but only suitable to applications where the depolarization times are of interest (since it does not calculate the repolarization phase of the action potential). Such an approach was used recently for patient-specific cardiac model personalization (Chinchapatnam et al 2008), where the authors used clinical EP measurements recorded by a basket electrode to perform customization of conductivity maps based on the conduction velocities calculated from measured activation depolarization times. Thus, while we agree that the Eikonal model is useful for customization of conductivity maps, it should be noted that the Aliev-Panfilov model allows more parameters to be customized (using patch-clamp or optical measurements). On the other side, the bidomain model is superior to the Aliev-Panfilov model, because it solves for a realistic, physical solution of the transmembrane potential, while the simplistic, macroscopic AlievPanfilov model gives only normalized values of the action potential wave. This is a limitation in accurately modelling ischemia (where the resting potential is about $-70 \mathrm{mV}$, compared to $-90 \mathrm{mV}$ for healthy tissue) and drug-tissue interaction. However, we preferred using the simplistic AlievPanfilov model over the bidomain model, because: i) from the experimental point of view, the recorded optical action potentials do not have absolute values for intra- and extracellular voltages, thus the bidomain predictions for $\mathrm{V}_{\text {ext }}$ and $\mathrm{V}_{\text {int }}$ cannot be validated from optical measures; and ii) from the computational point of view, the bidomain model requires solving a set of PDE for the direct solution. Computing 3D numeric solutions to this system is time consuming because of the large sparse linear systems, at each time step (even with an explicit time-stepping method), and also because of the time scale and the space scale (thus, the need of super-computers and clusters). Advantageously, the monodomain model is reduced to ordinary differential equations (ODE). These equations, in the phenomenological simplified model proposed by Aliev and Panfilov, can be solved 500-1000 time faster than a PDE. Moreover, Regarding the CPU times and performance of the model, the time step we used in the direct model is $5 \times 10^{-5} \mathrm{~s}$ (on meshes with average length of elements of $\sim 1.2 \mathrm{~mm}$ ); this resulted in a total computation time of $25 \mathrm{~min}$ for $0.8 \mathrm{~s}$ of heart cycle on a regular PC (i.e., Intel ® Core (TM) 2 Duo CPU $1.83 \mathrm{GHz}$, with 4GB of RAM), compared to $2 \mathrm{~h}$ for $1 \mathrm{~s}$ of heart cycle simulated with the Luo-Rudy model using thirty $1.4 \mathrm{GHz}$ processors in parallel (Xie et al., 2004) or 2 days with the bidomain model (Potse et al., 2006) on 32 processors. The latter study demonstrated that the propagations of the action potential (AP) were only $2 \%$ faster in the bidomain model than in the monodomain model, which also justifies our choice of model. In addition, for the direct solution of AP, the code used in this paper is relatively fast because we set $\varepsilon=0.01$ (where $\varepsilon^{-1}$ is a time constant associated to the recovery phase) as in the paper from Nash and Panfilov (2004); however, a more realistic value would be $\varepsilon=0.001$ (to be 
closer to the cell-membrane/unit volume), but such a small value needs a much finer mesh (likely 10 times smaller in each direction, which means 1000 time more unknowns in 3D, and a step 100 times smaller as well). Regarding the model performance for the inverse problem: the solution for each conductivity zone was derived in approx 15 min per zone on a regular PC (same as the one used in Relan et al 2011), resulting in a total computational time of approximately $6 \mathrm{~h}$ for the RF heart (for 26 zones), and approximately $12 \mathrm{~h}$ for the infarct heart (which had 52 zones).

In this paper, we report successful mapping of the AP measured from the epicardium of large pathologic hearts in a porcine model, ex vivo. Two relevant types of scars generating disturbed propagation in the presence of unexcitable areas were used in this experimental model: acute lesions created via RF thermal damage and chronic myocardial infarct scar. The mean APD of the waves optically recorded at low pacing frequency $(1.1 \mathrm{~Hz})$ in all these pathologic hearts were close to the mean values reported by us previously in healthy porcine hearts (Pop et al 2009a) and by other researchers (Caldwell et al 2005). The electrophysiological characteristics of the optical AP immediately following RF lesion creation ex vivo, revealed that the AP wave had no significant morphological changes in the very thin transitional area (called border zone, BZ) between healthy tissue (NZ) and RF scar (where, abruptly, no optical APs were recorded). The mean APD90 in the BZ (over three specimens) was reduced by only 4\% compared to APD90 in the NZ, and the AP wave had a barely distinguishable change in steepness for the recovery slope. As demonstrated by histology, this BZ zone surrounded the scar and contained the following: surviving myocytes with a healthy structural appearance and preserved organization; some small collapsed vessels; mild inflammation; and edematous fluid. All of these are typically found in the red rim of the acute phase of RF thermal damage. Thus, most likely, the small change in APD could be explained by the fact that, in the BZ of RF lesion, the ionic concentration changes in the extracellular space due to fluid accumulation (produced by an outward 'drift' of water, from the core of the RF scar toward its margin, during dessication). Furthermore, several cells (contributing to the optical signal from the pixels in the BZ) could undergo acute ischemia, which always triggers a shorter APD.

On the other hand, the relative changes in APD in the BZ of the myocardial infarcts were found to be as large as $14 \%$. The shortening of the APD in this area was consistently observable 4 weeks after infarction in the occlusion-reperfusion model in all 3 hearts we studied. This is in contrast with another optical study performed in a non-reperfused rat model of MI 7 days after infarction which did not find significant changes in APDs in the border zone; however, this could be due to the infarction model. That study also demonstrated that APs in the BZ have lower 
amplitude and slower up-stroke (potentially due to a reduction in the peak sodium current), which is in accordance to our observations. Meanwhile, a longitudinal study reported that the shortest APD measured in canine myocardial cells is after 2 weeks and full recovery of APD was observed at 2 months using canine infarcts (Ursell et al 1996). Our measurements fall between this range, with values that are shorter than normal at week 4 .

M-cells (typically found in the mid-wall) are known to exhibit longer APDs in larger hearts, from pigs weighing 60-80kg (Stankovicova et al 1999) and other species, including humans (Janse and Wit, 1989). Our APD recordings from optical fluorescence measurements were limited to the epicardium; however, we do not expect significant transmural variation in our model because it was demonstrated via intramural optrode measurements that juvenile pigs (of similar weight to our study) lack M-cells (Caldwell et al 2005). From the modelling perspective, the fact that there is no transmural heterogeneity in the APD for juvenile pigs means that we correctly used the same $a$-values in the NZ across the wall; this was valid for both models (regardless of the scar type).

The restitution curves derived in our studies from recordings at different frequencies were as expected, with slopes <1 (for normal myocardium). More importantly, for NZ, the simulated restitution curve was produced after customizing the parameter ' $a$ ' in the model with the corresponding value estimated from measurements. We used the average value of ' $a$ ' and compared the resulting curve to two experimental curves; the simulated curve behaved rather well suggesting that it could be used to generically represent the memory effects in APD in normal porcine myocardium, at least in the case of dynamic pacing (constant cycle length). We acknowledge that it is difficult to customize simultaneously $d, a$ and $k$ since they actually depend on each other (for instance, the up-stroke of the AP wave can be change by manipulating either $k$ or $d$ parameter). With this respect, the Mitchell-Schaeffer model, for instance, is a superior model to use since $a$ and $d$ can be fitted independently, as recently demonstrated in a study performed in healthy hearts (Relan et al 2011). However, we suggest that the following pairs $(a=0.112, k=8)$ and ( $a=2, k=2)$ could be generically used as input to this particular model, to reproduce the characteristics of the AP wave in healthy and ischemic BZ areas, respectively, for porcine hearts with 4-5 week old infarction. For $d$, one has to consider a certain scaling due to the size of the heart and element size, but generally values of $d=2$ (or 3 ) could be used for healthy tissue, while reduced values should be used to represent the conductivity in the $\mathrm{BZ}$ of such chronic cases. 
The values for conduction velocities measured in the NZ, and consequently the anisotropy ratio computed in our study, are within the normal range measured and reported by other investigators in pig hearts and are close to values reported in rabbit, rat and human hearts under paced conditions in explanted hearts (Janse and Wit 1989, Sung et al 2000). As a limitation, we did not measure the velocity ratio in the BZ, which could be different than the $\sim 1: 2.7$ ratio we obtained in normal myocardial tissue. Such specific values were found to be different in rat models (Mills et al 2006) and human hearts (De Bakker et al 1988, Kawara et al 2001); these human studies reported a conduction velocity in the transverse direction severely reduced due to fibrosis deposition in the substrate area. Thus, the anisotropy ratio in the BZ could have an impact from the model's perspective; however, the latter human measurements were taken in patients with heart failure, years after infarction. Further experimentation is needed with this respect, with smaller FOV and focusing on BZ areas, rather than mapping the whole large heart.

Overall, the differences in electrophysiological measurements across regions corresponded very well with the varying myocardial characterization determined from MR images and histology. The very thin BZ in the RF scar model was not included as a separate zone; thus this model was simplified to a two-zone model that accurately predicted the propagation of the AP wave in the presence of a large unexcitable scar. The MI model has three MR-image-based zones (whose extent was validated against histology with good agreement).

The other achievement of this paper is that we successfully built a realistic macroscopic 3D cardiac computer model of pathologic hearts and performed 3D volumetric adjustment of conductivity maps. The construction of each 3D computer model from MR images was based on integrating information of scar morphology and of structural anisotropy from MR measurements of fiber directions. The model customization and $3 \mathrm{D}$ volumetric adjustment were successfully performed; for example, from one pacing location, the adjustment reduced the absolute error in local depolarization times by a factor of 5 (i.e., from $58 \mathrm{~ms}$ to $11 \mathrm{~ms}$ ) in the infarcted heart, and by a factor of 6 (i.e., from $\sim 60 \mathrm{~ms}$ to $9 \mathrm{~ms}$ ) in the heart with the RF scar. Moreover, the sensitivity of adjusted conductivity maps to different pacing locations was tested, and the errors in activation times were found to be of approximately 10-12 ms, suggesting that any location can be used for model predictions.

Lastly, we studied the impact of perturbation in fiber directions on the wave propagation. This was accomplished by generating synthetic fibers datasets with helix angle allowed to vary transmurally between the following ranges (from the endocardium to the epicardium): $-50 \%+50^{\circ}$, 
$-60^{\circ} /+60^{\circ},-70^{\circ} /+70^{\circ},-80^{\circ} \%+80^{\circ}$ and $-90^{\circ} \%+90^{\circ}$. Our results showed that the comparison between the activation times obtained using these synthetic fibers datasets and DTI fibers, yielded good correlation and small error for the datasets with the helix angles varying from $+/-60^{\circ},+/-70^{\circ}$ and $+/-80^{\circ}$. These datasets had the smallest RMS error and mean \pm S.D associated with, and very good correlation coefficients (e.g. 0.94 for the RF heart and 0.82 for the infarcted heart from one of the pacing locations for the $+/-70^{\circ}$ synthetic dataset). We therefore suggest that synthetic fibers can be used within an acceptable margin of error for the activation times, when DTI data is not available. This result is also important for applications aiming to translate such 3D MRI-based models in the clinics, since DTI is not used routinely in-vivo due to motion artifacts (Wu et al 2006).

In summary, our results demonstrated overall that a simple mathematical model of electrophysiology (Aliev-Panfilov model) can be customized using optical measurements of AP as well as MR images acquired in pathologic hearts. Several parameters can be directly estimated from 2D optical action potential measurements ( $a, k$, anisotropy ratio); in addition 3D volumetric adjustment of local conductivity is feasible for both models built from pathologic hearts (i.e., with large conduction blocks RF acute lesion and infarct scar). Regarding 3D model customization from measurements, we will address the parameter estimation step using more complex and biophysical models. Such efforts are ongoing and preliminary results were reported recently for healthy heart models, showing that the error in activation time converges faster using models of intermediate complexity, and that more parameters corresponding to the APD and conduction velocity restitution curves can be adjusted independently (Relan et al 2011).

\section{Limitations of the $3 D$ computer model}

Although we obtained good correspondence between measurements and simulations after the customization step, our simple approach using a macroscopic formalism might not be accurate enough compared to complex biophysical models. However, one related issue is the spatial scale and mesh density used in computational studies. With this respect, it was recently shown in a wide benchmark study (Niederer et al 2011) that for complex biophysical models (e.g. Ten Tusscher \& co model) only very fine meshes (sub-milimetric resolution) can be used to obtain accurate solution and correct numerically convergence. In our study, we approached the monodomain model with a 'macroscopic formalism' at tissue level and not at the cellular level; thus, we used meshes with mean element size of 1.3 and $1.5 \mathrm{~mm}$. We acknowledge that this mesh resolution could introduce a small error in the computation of upstroke and conduction velocity of 
wavefront. Therefore, to provide justification for our meshes used, we performed an additional convergence test in which we computed the conduction velocity on a virtual slab of tissue $(1 \times 1 \times 3 \mathrm{~cm})$, using the Aliev-Panfilov model and different mesh resolutions (i.e., element edge size of: $5 \mathrm{~mm}, 3.33 \mathrm{~mm}, 2.5 \mathrm{~mm}, 2 \mathrm{~mm}, 1 \mathrm{~mm}, 0.5 \mathrm{~mm}$ and $0.33 \mathrm{~mm}$, respectively). In Figure 16, we plotted the mean conduction velocity at ten different points (as the mesh is refined).

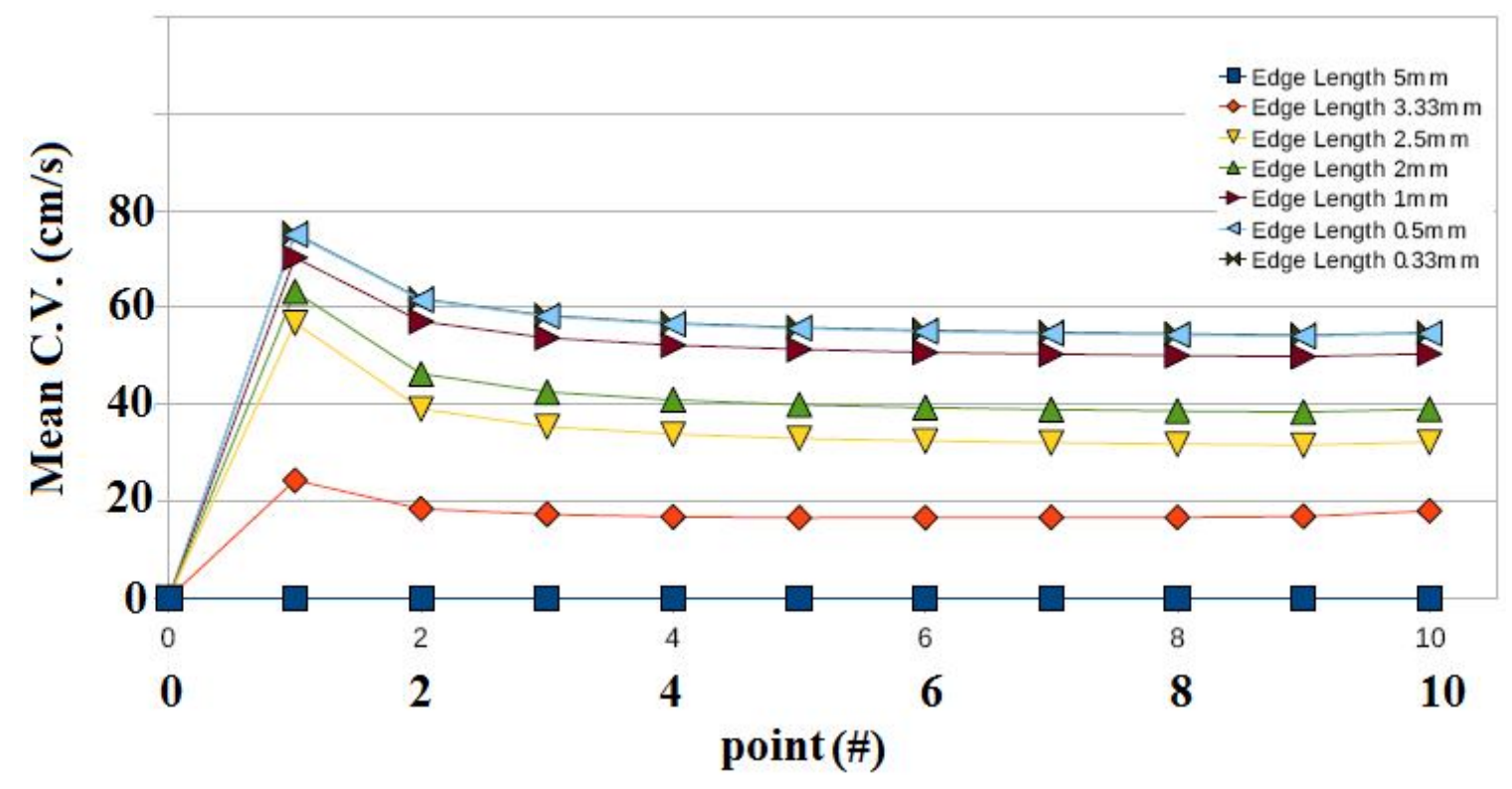

Fig. 16 Convergence test for mean conduction velocity, C.V. (cm/s), computed from activation times for different mesh resolutions (values displayed at ten random different points on the slab)

The convergence test demonstrated that meshes with element edge less than $2 \mathrm{~mm}$ but close to 1 mm slightly underestimate the $\mathrm{CV}$ compared to denser meshes $(0.5 \mathrm{~mm}$ or finer resolution); thus our future work will address in detail this particular problem and the spatial-scale effect on the $d$ maps using realistic different 3D heart models (healthy and pathologic). Notable, a potential solution could be the use of the recent pipeline proposed by Camara et al (2011) (which is based on the optical and MRI data from Pop et al 2009b), where the authors demonstrated the advantages and disadvantages of several simple and complex models, and showed that these models can also be integrated into the same pipeline. 


\section{Conclusion}

In this work, we built a 3D cardiac model of pathology and parameterized the model using a simple monodomain macroscopic model of cardiac electrophysiology in pathological hearts. Specifically, we compared the output of a computer model calibrated with MRI data (depicting anatomy, scar heterogeneity and fiber orientations), with measurements of action potential obtained using an optical imaging technique in large, porcine hearts with scars. Further analysis of AP wave characteristics (i.e., up-stroke, duration and restitution effects at different pacing frequencies) and the speed of the propagation of the depolarization wavefront can be used to adjust locally the model input parameters in heterogeneous myocardium tissue (i.e., healthy and border zone).

\section{Acknowledgements}

The author would like to thank: Mr. Desmond Chung for developing the stereoscopy and registration codes, the veterinary technicians at Sunnybrook Health Sciences Centre (Toronto) who helped during the optical and infarction experiments, as well as Mrs Lily Morikawa and Ms Lisa Dang for help with histological staining and scanning. Dr. Mihaela Pop was supported by a research award from Heart and Stroke Foundation of Canada, and an OGSST scholarship. The research work received support by funding from the Canadian Institutes of Health Research (Grant Number MOP93531). 


\section{Supplemental Material}

Table 1 Error analysis quantifying the differences between activation times obtained from simulations using DTI fibers and simulations using different synthetic fibers, respectively. Included are: RMS error (\%), Pearson correlation coefficient (r) and the mean \pm S.D. (ms) for these differences in activation times, respectively (calculated for each heart and each pacing location).

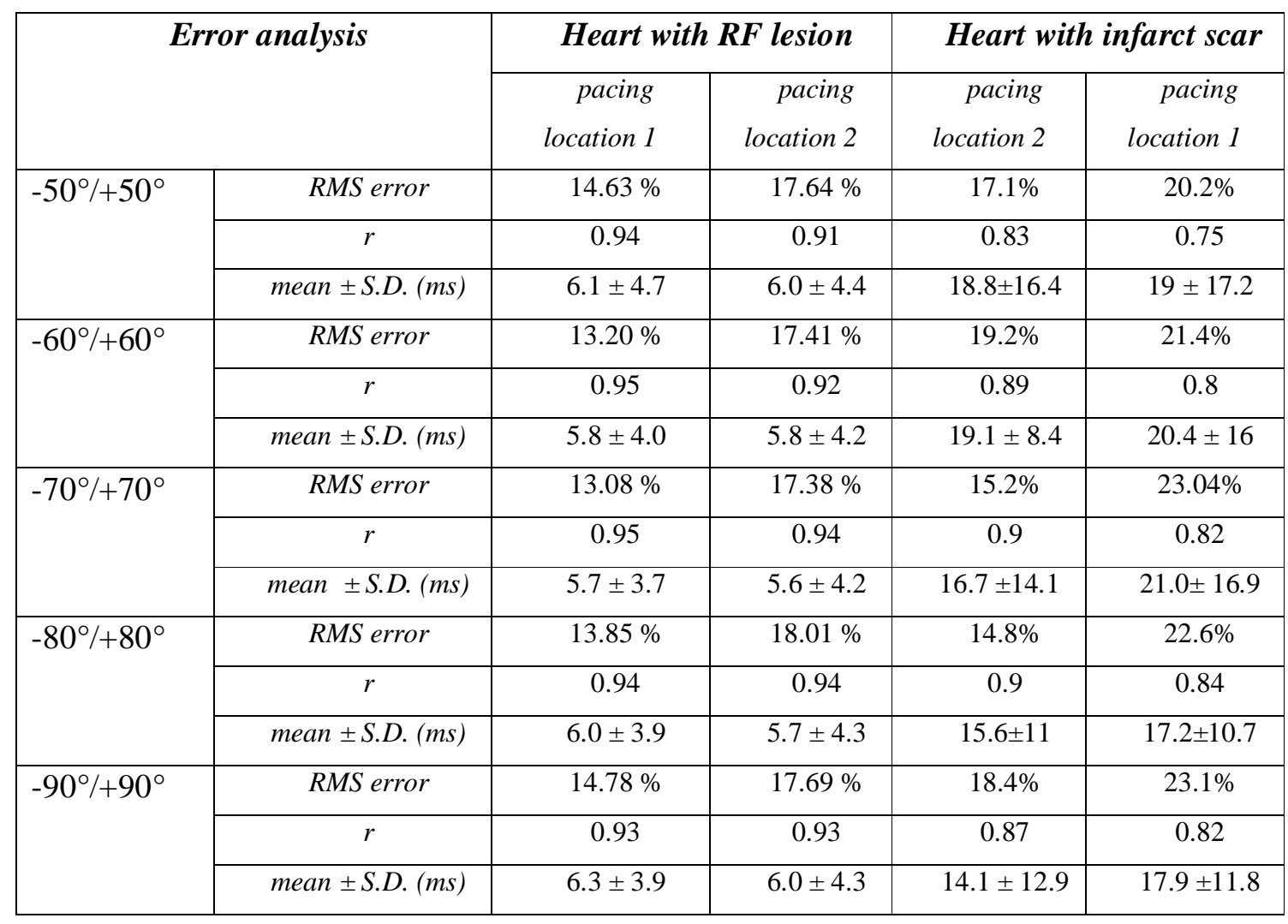




\section{References:}

Aliev R. R. and Panfilov A. V. (1996a) Modeling of heart excitation patterns caused by a local inhomogeneity Journal of theoretical biology 181, 33-40.

Aliev R. R. and Panfilov A. V. (1996b) A Simple Two-variable Model of Cardiac Excitation Chaos, Solitons and Fractals 7, 293-301.

Ashikaga H, Sasano T, Dong J et al., (2007) Magnetic resonance-based anatomical analysis of scar-related ventricular tachycardia: implications for catheter ablation, Circulation Research, 101 (9), 939-947

Austin T, Trew M, Pullan A (2006) Solving the cardiac bidomain equations for discontinuous conductivities IEEE Trans on Biomed Eng 53 (7) 1265-1272

Banville I., and Gray R. A. (2002) Effect of action potential duration and conduction velocity restitution and their spatial dispersion on alternans and the stability of arrhythmias, J Cardiovasc Electrophysiol 13, 1141-1149.

Bayly P. V., KenKnight B. H., Rogers J. M., Hillsley R. E., Ideker R. E., and Smith W.M. (1998). Estimation of conduction velocity vector fields from epicardial mapping data, IEEE Transactions on Biomedical Engineering 45, 563-571.

Bishop M, Plank G, Burton R.A.B, Schneider J.E., Gavaghan D.J., Grau V, Kohl P (2009) Development of an anatomically detailed MRI-derived rabbit ventricular model and assessment of its impact on simulations of electrophysiological function Am J Physiol Heart Circ Physiol 298: H699-H718

Camara O, Sermesant M, Lamata P, Wang L, Pop M, Relan J, De Craene M, Delingette H, Liu H, Niederer S, Plank G, Pashaei A, Romero D, Sebastian R, Wong KCL, Zhang H, Ayache N, Frangi A, Shi P, Smith NP, Wright GA; Inter-model consistency and complementarities: learning from ex-vivo imaging and electrophysiological data towards an integrated understanding of cardiac physiology; Prog. Biophys. Molec. Biol. 107; 122-133.

Cerqueira, M. D., Weissman, N. J., Dilsizian, V., Jacobs, A. K., Kaul, S., Laskey, W. K., Pennell, D. J., Rumberger, J. A., Ryan, T., and Verani, M. S. (2002). Standardized myocardial segmentation and nomenclature for tomographic imaging of the heart: a statement for healthcare professionals from the Cardiac Imaging Committee of the Council on Clinical Cardiology of the American Heart Association, Circulation 105, 539-542.

Caldwell B.J., Legrice I.J., Hooks D.A., Tai D.C-S., Pullan A.J., and Smaill B.H. (2005) Intramural measurement of transmembrane potential in the isolated pig heart: Validation of a novel technique, J. Cardio. Electrophys., 16(9), 1001-1010 
Chinchapatnam P, Rhode KS, Ginks M, Rinaldi CA, Lambiase P, Razavi R, Arridge S, and Sermesant M. (2008) Model-Based Imaging of Cardiac Apparent Conductivity and Local Conduction Velocity for Diagnosis and Planning of Therapy. IEEE Transactions on Medical Imaging, 27(11):1631-1642

Chung D., Pop M., Sermesant M., and Wright G. A. (2006) "Stereo reconstruction of the epicardium for optical fluorescence imaging," in MICCAI Workshop on Biophotonics for diagnosis and treatment. (Editors BJ Esboll \& TM Jorgensen), pp. 33-40.

Clayton R H (2001) Computational models of normal and abnormal action potential propagation in cardiac tissue: linking experimental and clinical cardiology Physiol. Meas. 22 R15-R34

Clayton RH and Panfilov AV (2008) A guide to modelling cardiac electrical activity in anatomically detailed ventricles Prog Biophys Mol Biol 96 (1-3) 19-43

Conn A.R., Gould N.I.M, Toint Ph. L.: (2000) Trust region methods, MPS-SIAM Series on Optimization 1, SIAM, Philadelphia, USA (ISBN-13: 978-0-898714-60-9)

De Bakker JMT, Van Capelle FJL, Janse MJ, Wilde AAM, Coronel R, Becker AE, Dingemans KP, Van Hemel NM , Hauer RNW (1988) Reentry as a cause of VT in patients with chronic ischemic heart disease: electrophysiologic and anatomic correlation Circulation, 77(3) 589-606

Dukkipati S, Mallozi R, et al (2008) Electroanatomic mapping of the left ventricle in a porcine model of chronic myocardial infarction with magnetic resonance-based catheter tracking Circulation;118(8):853-62.

Efimov I. R., Huang T.D., Rendt J.M, and Salama G. (1994) Optical mapping of repolarization and refractoriness from intact heart, Circulation research 90, 1469-1480.

Efimov I. R., Nikolski V. P. and Salama, G. (2004) Optical imaging of the heart, Circulation research 95, 21-33.

Elharrar V., and Surawicz B. (1983) "Cycle length effect on restitution of action potential duration in dog cardiac fibers," The American journal of physiology 244, H782-792.

Eldar M., D. G. Ohad, J. J. Goldberger, Z. Rotstein, S. Hsu, D. K.Swanson, and A. J. Greenspon, "Transcutaneous multielectrode basket catheter for endocardial mapping and ablation of ventricular tachycardia in the pig," Circulation, vol. 96, no. 7, pp. 2430-2437, 1997.

Faris O.P., Evans F.J., Ennis DB, Helm PA, Tylor JL, Chesnick AS, Guttman MA, Ozturk C and McVeigh ER (2003) "Novel technique for cardiac electro-mechanical mapping with MRI tagging and epicardial sock" Am. Journal of Physiology. Heart and Circ. Physiology, 285(5): H1864-70.

Gavaghan D, Garny A, Maini PK, Kohl P. (2006) Mathematical models in physiology. Philos Transact A Math Phys Eng Sci. May 15;364(1842):1099-106

Girouard SD and Rosenbaum DS (2001) Role of wavelength adaptation in the initiation, maintenance, and pharmacologic suppression of reentry J. Cardiovasc. Electrophysiology 12(6): 697-707 
Hao S. C., Christini D. J., Stein K. M., Jordan P. N., Iwai S., Bramwell O., Markowitz S. M., Mittal S., and Lerman B. B. (2004). Effect of beta-adrenergic blockade on dynamic electrical restitution in vivo, American journal of physiology 287, H390-394.

Helm P. A., Tseng H. J., Younes L., McVeigh E. R., and Winslow R. L. (2005). "Ex vivo 3D diffusion tensor imaging and quantification of cardiac laminar structure, Magn Reson Med 54, 850-859.

Hillman E. M. C., Bernus O., Pease E., Bouchard M. B., and Pertsov A. (2007). Depth-resolved optical imaging of transmural electrical propagation in perfused heart Optics Express 15, 17827-17841.

Horn B. K. P. (1987) Closed-form solution of absolute orientation using unit quaternions Journal of the Optical Society of America A 4, 629-642.

Hunter PJ, Crampin EJ, Nielsen PM. (2008) Bioinformatics, multiscale modeling and the IUPS Physiome Project. Brief Bioinform. Jul; 9 (4): 333-43.

Hunter PJ and Nielsen PM (2005) A strategy for computational integrative physiology Physiology 20: 316325

Hyatt C. J., Mironov S. F., Vetter F. J., Zemlin C. W., and Pertsov A. M. (2005a) Optical action potential upstroke morphology reveals near-surface transmural propagation direction Circulation Research 97, 277-284.

Hyatt C. J., Mironov S. F., Vetter F. J., Zemlin C. W., and Pertsov A. M. (2005b) Optical action potential upstroke morphology reveals near-surface transmural propagation direction Circulation research 97, 277-284.

Janse MJ and Wit AL (1989) Electrophysiological mechanisms of ventricular arrhythmias resulting from myocardial ischemia and infarction Physiological Reviews, 69 (4), 1049-1169.

Kadish A., Shinnar M., Moore E. N., Levine J. H., Balke C. W., and Spear J. F. (1988) Interaction of fiber orientation and direction of impulse propagation with anatomic barriers in anisotropic canine myocardium Circulation 78, 1478-1494.

Kay M. W., Amison P. M. and Rogers J. M. (2004) Three-dimensional surface reconstruction and panoramic optical mapping of large hearts IEEE Transactions on Biomedical Engineering 51, 1219-1229.

Kawara T, Derksen R, de Groot J R, Coronel R, Tasseron S, Linnenbank AC, Hauer R N, Kirkels H, Janse M J, de Bakker J M T Activation delay after premature stimulation in chronically diseased human myocardium relates to the architecture of interstitial fibrosis Circulation 104 3069-3074, 2001

Kleber AG and Rudy Y (2004) Basic mechanisms of cardiac impulse propagation and associated arrhythmias Physiological Review 84, 431-488

Keener J.P. and Sneyd J, (1998) Mathematical physiology, Spinger 
Kim B. S., Kim Y. H., Hwang G. S., Pak H. N., Lee S. C., Shim W. J., Oh D. J., and Ro Y. M. (2002) Action potential duration restitution kinetics in human atrial fibrillation Journal of the American College of Cardiology 39, 1329-1336.

Kleber A. G. and Rudy Y. (2004). "Basic mechanisms of cardiac impulse propagation and associated arrhythmias," Physiological reviews 84, 431-488.

Van Leemput K, Maes K , F. Vandermeulen F, Colchester A, Suetens P (2001) Automated segmentation of multiple sclerosis lesions by model outlier detection. IEEE Trans. on Medical Imaging. vol.20, no.8, 677-688

Lepiller D, Sermesant M, Pop M, Delingette H, Wright GA, Ayache N (2008) Cardiac electrophysiology model adjustment using the fusion of MR and optical imaging In Dimitris N. Metaxas, Leon Axel, Gabor Fichtinger, and Gábor Székely, editors, Proc. Medical Image Computing and Computer Assisted Intervention (MICCAI'08), volume 5241 of Lecture Notes in Computer Science, pages 678-685, 2008. Springer

Li L, Nikolski V, Wallick D, Efimov I, and Cheng Y. (2003) Redistribution of Cx43 expression in the endocardial infarction border zone is associated with increased arrhythmogenesis in the rabbit heart Circulation 108: IV-189

Martinez-Rubio A, Bayes-Genis A, Guindo J, Bayes de Luna A (1999) Sudden cardiac death Contributions to Science 1 147-157,

Mills WR, Mal N, Forudi F, Popovic ZB, Penn MS, Laurita KR (2005) Optical mapping of late myocardial infarctions in rats Am J Heart Circ Physiol (290) H1298-1306

Moreau-Villeger V., Delingette H., Sermesant M., Ashikaga H., McVeigh E., and Ayache N. (2006) Building maps of local apparent conductivity of the epicardium with a 2-D electrophysiological model of the heart IEEE transactions on bio-medical engineering 53, 1457-1466.

Muzikant A.L. and Henriquez C.S. (1998): Validation of three-dimensional conduction models using experimental mapping: are we getting closer? Prog Biophys Mol Biol. 1998;69(2-3):205-23.

Nash M. P., and Panfilov A. V. (2004). "Electromechanical model of excitable tissue to study reentrant cardiac arrhythmias," Progress in biophysics and molecular biology 85, 501-522.

Nanthakumar K., Jalife J., Masse S., Downar E., Pop M., Asta J., Ross H., Rao V., Mironov S., Sevaptsidis E., Rogers J., Wright G., and Dhopeshwarkar R. (2007) Optical mapping of Langendorff-perfused human hearts: establishing a model for the study of ventricular fibrillation in humans American Journal of Physiology-Heart and Circulatory Physiology 293, H875-H880.

Niederer S, Kerfoot E, Benson AP, Barnabeu MO, Bernus O, Bradley C, Cherry EM, Clayton R, Fenton FH, Garny A, Heidenreich E, Land S, Maleckar M, Pathmanathan P, Plank G, Rodriguez JF, Roy I, Sachse FB, Seemann G, Skavhaug O, Smith NP (2011) Verification of cardiac tissue electrophysiology simulators using an N-version benchmark Phils. Trans. Royal Society 369; 4331-4351 
Qin H, Kay MW, Chattipakon N, Redden D.T., Ideker RE, Rogers JM (2003) Effects of heart isolation, voltage-sensitive dye, and electromechanical uncoupling agents on ventricular fibrillation Am. Journal of Physiology. Heart and circ. Physiology, 284: H1818-H1826.

Qu F, Nikolski V, Efimov I, Deng C (2004) Fluorescent real-time monitoring of HIFU cardiac focal ablation J. Acoust. Soc. Am. Volume 115, Issue 5, pp. 2448-2448

Pastore, J. M., and Rosenbaum, D. S. (2000). Role of structural barriers in the mechanism of alternansinduced reentry, Circulation Research 87, 1157-1163.

Pop M, Sermesant M, Chung D, Liu G, McVeigh ER, Crystal E, and Wright GA. (2007) An experimental framework to validate 3D models of cardiac electrophysiology via optical imaging and MRI. In Proceedings of Functional Imaging and Modeling of the Heart 2007 (FIMH'07), volume 4466 of LNCS, pages 100-109, 7-9 June

Pop M, Sermesant M, Lepiller D, Truong MV, McVeigh ER, Crystal E, Dick AJ, Delingete H, Ayache N, Wright GA (2009a) Fusion of optical imaging and MRI for the evaluation and adjustment of macroscopic models of cardiac electrophysiology: a feasibility study Medical Image Analysis Apr; 13(2): $370-80$

Pop M, Sermesant M, Mansi T, Crystal E, Detsky JS, Yang Y, Fefer P, McVeigh ER, Dick AJ, Ayache N, Wright GA (2009b) Characterization of porcine infarcts heart: insights from experiments and theoretical modelling in Proceedings of the FIMH, June 3-5 2009, Nice - LNCS vol 5528: 1-10, (Springer-Verlag Berlin Heidelberg).

Potse M, Dube B, Richer J, Vinet A, Gulrajani RM (2006) A comparison of monodomain and bidomain reaction-diffusion models for action potential propagation in human heart IEEE Transactions in Biomedical Engineering 53 2425-35

Prassl A.J., F. Kickinger, H. Ahammer, V. Grau, J.E. Schneider, E. Hofer, E.J. Vigmond, N.A. Trayanova, G. Plank (2009) Automatically generated, anatomically accurate meshes for cardiac electrophysiology problems IEEE Trans Biomed Eng., 56(5): 1318-30.

Relan J, Sermesant M, Delingette, Pop M, Sorine M, Wright GA, Ayache N. (2009a) Parameter Estimation of a 3D Cardiac Electrophysiology Model Including the Restitution Curve Using Optical and MR Data. In Olaf Dössel and Wolfgang C. Schlegel, editors: World Congress on Medical Physics and Biomedical Engineering, volume 25/IV of IFMBE Proceedings, Munich, Germany, 1716-1719 (Springer)

Relan J, Sermesant M, Pop M, Delingette H, Sorine M, Wright GA, Ayache N. (2009b) Volumetric Prediction of Cardiac Electrophysiology using a Heart Model Personalised to Surface Data. In MICCAI Workshop on Cardiovascular Interventional Imaging and Biophysical Modelling CI2BM09, London, UK, 19-27. 
Relan J, Pop M, Delingette H, Wright GA, Ayach N, and Sermesant M (2011) Personalisation of a Cardiac Electrophysiology Model using Optical Mapping and MRI for Prediction of Changes with Pacing. IEEE Transactions on Biomedical Engineering (in press, e-pub 20 January 2011)

Schmitt C., Zrenner B., Schneider M, Karch M, Ndrepepa G, Deisenhofer I, Weyerbrock S, Schreieck J, and Schomig A, "Clinical experience with a novel multielectrode basket catheter in right atrial tachycardias", Circulation, vol. 99, no. 18, pp. 2414-2422, 1999.

Schmidt A, Azevedo CF, Cheng A et al., (2007) Infarct tissue heterogeneity by magnetic resonance imaging identifies enhanced cardiac arrhythmia susceptibility in patients with left ventricular dysfunction," Circulation, 115 (15), 2006-2014.

Sermesant M., Delingette H., and Ayache N. (2006). "An electromechanical model of the heart for image analysis and simulation," IEEE transactions on medical imaging 25, 612-625.

Sermesant M., Faris, O., Evans F., McVeigh ER., Coudiere Y., Delingette H., and Ayache N. (2003). "Preliminary Validation Using in vivo Measures of a Macroscopic Electrical Model of the Heart" in Surgery Simulation and soft Tissue Modelling (Springer Berlin / Heidelberg).

Sermesant M., Rhode K., Sanchez-Ortiz G. I., Camara O., Andriantsimiavona R., Hegde S., Rueckert D., Lambiase P., Bucknall C., Rosenthal E., Delingette H., Hill D. L., Ayache N., and Razavi R. (2005). "Simulation of cardiac pathologies using an electromechanical biventricular model and XMR interventional imaging," Medical image analysis 9, 467-480.

Stankovicova T, Szilard M, De Scheerder I, Sipido KR (2000) M cells and transmural heterogeneity of action potential configuration in myocytes from the left ventricular wall of the pig heart Cardiovasc Research 45, 952-960

Sung D., Omens J. H., and McCulloch A. D. (2000) Model-based analysis of optically mapped epicardial activation patterns and conduction velocity Annals of biomedical engineering 28, 1085-1092.

Taccardi B, Punske BB, Macchi E, Macleod RS, Ershler PR (2008) Epicardial and intramural excitation during ventricular pacing: effect of myocardial structure Am. Journal of Physiology. Heart and circ. Physiology, 294 (4) H1753-66

Ten Tusscher KHWJ and Panfilov AV (2007) Influence of diffuse fibrosis on wave propagation in human ventricular tissue Europace 9; 38-45

Vetter F. J., Simons S. B., Mironov S., Hyatt C. J., and Pertsov A. M. (2005). Epicardial fiber organization in swine right ventricle and its impact on propagation Circ research 96, 244-251.

Trayanova N (2006) Defibrillation of the heart: Insights into mechanism from modelling studies Experimental Physiology 91 323-327

Trayanova N (2009) In the spotlight: Cardiovascular Engineering IEEE Rev Biomed Engineering Nov 17; 2:12 
Vadakkumpadan F, Rantner L, Tice B, Boyle P, Prassl A, Vigmond E, Plank G, Trayanova N. (2009) Image-based models of cardiac structure with applications in arrhythmia and defibrillation studies, J. Electrocardiology 42 (2) 157

Vadakkumpadan F, Arevalo H, Prassl AJ, Chen J, Kickinger F, Kohl P, Plank G, Trayanova N (e-pub ahead of print 2010) Image-based models of cardiac structure in health and disease (in press in WIREs Syst Biol Med (Willey InterScience)

Vigmond E, Vadakkuumpadan D, Gurev V, Deo M, Plank G, Trayanova N (2009) Towards predictive modelling of the electrophysiology of the heart Experimental Physiology 94 562-77

Walton MK and Fozzard HA (1983) The conducted action potential: models and comparison to experiments Biophysical Journal 44: 9-26

Wu M. T., Tseng W. Y. I., Su M. Y. M., Liu C. P., Chiou K. R., Wedeen V. J., Reese T. G., and Yang C. F. (2006). "Diffusion tensor magnetic resonance imaging mapping the fiber architecture remodeling in human myocardium after infarction - Correlation with viability and wall motion," Circulation 114, 1036-1045.

Wu EX, Wu Y, Nicholls JM et al., (2007) MR diffusion tensor imaging study of postinfarct myocardium structural remodeling in a porcine model," Magn Reson Med, 58 (4), 687-695.

Yan AT, Shayne AJ, Brown KA et al., (2006) Characterization of the peri-infarct zone by contrastenhanced cardiac magnetic resonance imaging is a powerful predictor of post-myocardial infarction mortality Circulation, 114 (1), 32-39

Yang Z., Zhang H., Kong S., Yue X. F., Jin Y. B., Jin J., and Huang Y. C. (2007). "Study for relevance of the acute myocardial ischemia to arrhythmia by the optical mapping method," Physiological Measurement 28, 481-488. 